\title{
Nonlinear Control for the Dual Smart Drive Using Backstepping and a Time-Optimal Reference
}

\author{
Roemi Fernández ${ }^{1 *}$, João Hespanha ${ }^{2 \dagger}$, Teodor Akinfiev ${ }^{1 \ddagger}$ and \\ Manuel Armada ${ }^{1}$ \\ ${ }^{1}$ IAI/CSIC-Industrial Automation Institute, Spanish Council for Scientific \\ Research. Automatic Control Department. La Poveda 28500 Arganda del Rey, \\ Madrid, Spain. \\ ${ }^{2}$ CCEC/UCSB-Center for Control Engineering and Computation. University of \\ California, Santa Barbara, CA 93106-9560 USA.
}

\begin{abstract}
The Dual Smart Drive is a specially designed nonlinear actuator intended for use in climbing and walking legged robots. It features a continuously changing transmission ratio and dual properties and is very suitable for situations where the same drive is required to perform two different types of start-stop motions of a mobile link. Then, the associated control problem to this nonlinear actuator is established and a backstepping design strategy is adopted to develop Lyapunovbased nonlinear controllers that ensure asymptotic tracking of the desired laws of motion, which have been properly selected using time-optimal control. The approach is extended for bounded control inputs. Both simulation and experimental results are presented to show the effectiveness and feasibility of the proposed nonlinear control methods for the Dual Smart Drive.
\end{abstract}

Keywords: Dual Smart Drive, quasi-resonance drive, start-stop regime, nonlinear control, backstepping, time-optimal control, legged robots.

\section{Introduction}

In the current state of the art, legged robots' performance is characterized by very low speeds and high energy expenditure, resulting in low efficiency for these machines. This feature, which is due mainly to the use of conventional drives in actuators, considerably restricts the potential of legged robots (Armada et al., 2003) and limits their autonomous operating time. Selecting an appropriate drive system is, then, one of the most telling factors in overcoming the stated drawbacks (Pfeiffer et al., 2000), (Sardin et al., 1998). Conventional analysis of the walking process in legged robots distinguishes between two different phases in the locomotion cycle. The first phase takes place when all the

* Supported by the Spanish Ministry of Education under Grant F.P.U.

$\dagger$ Supported by the National Science Foundation under Grant No. ECS-0242798.

¥ Supported by the Spanish Ministry of Science and Technology under Grant Ramón y Cajal, Project "Theory of optimal Dual Drives for Automation and Robotics"

(C) 2005 Kluwer Academic Publishers. Printed in the Netherlands.

nRF-JAR-04-034.tex; 28/07/2005; 18:32; p.1 
legs are on the ground and the body is moving forward. The second one is the swing phase, where one or more legs are on the ground while the other(s) are swinging. A leg drive therefore works in two different regimes. First, it moves the heavy robot body in relation to the fixed leg. Then, the same drive moves the leg in relation to the fixed body. These two working regimes are quite different, because of the great difference between the body's mass and the mass of each of the legs (see Figures 1(a) and 1(b)). Thus, when a drive is tuned to one of the regimes (usually the first one, since that is the more taxing regime), it appears to be inefficient during the second regime, because it generally makes the leg movement too slowly, despite the high power of the drive motor. An additional difficulty with walking robots is that the motors need to operate in a start-stop mode, where traditional motors exhibit low efficiency (Chilikin and Sandler, 1981).

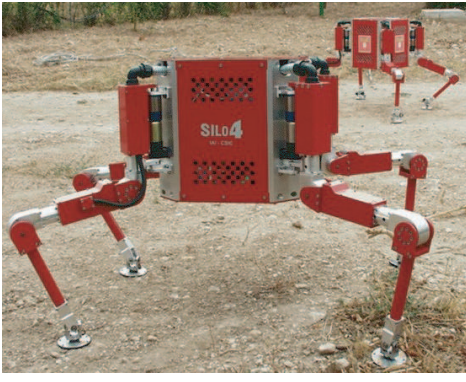

(a)

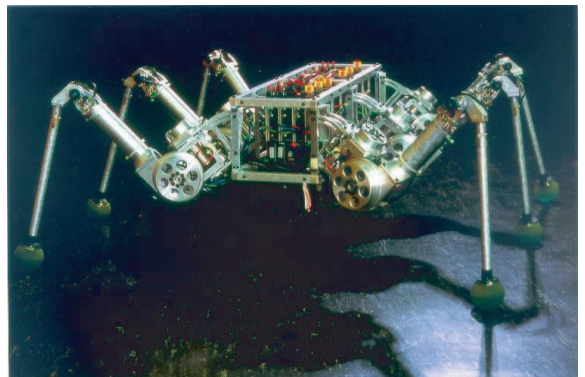

(b)

Figure 1. (a) The Silo-4 robot. (b) The six-legged TUM-Walking Machine.

All these considerations are sound reasons for developing new drives to provide practical solutions to the aforementioned problems. Several authors (Akinfiev et al., 1999), (Akinfiev and Armada, 2000), (Bruneau et al., 2000), (Budanov, 2001), (Caballero et al., 2001), (Ingvast and Wikander, 2002), (Roca et al., 2002), (Van De Straete and De Schutter, 1999) have demonstrated that using drives with some sort of variable reduction is a good way to increase robot efficiency. Some such drives (Bruneau et al., 2000), (Caballero et al., 2001), (Roca et al., 2002), are based on quasi-resonance drives (Akinfiev and Armada, 1998). They use variable geometry to accomplish variable reduction, and the arrangement yields different transmission ratios at different positions of the output joint. The quasi-resonance drive can thus be optimized for a specific task.

The drive described here, the Dual Smart Drive (Akinfiev et al., 2005), is also a further development of quasi-resonance drives, and it 
provides a continuously changing transmission ratio that depends on the angular position of the mobile link. The drive consists of a DC motor coupled with a constant transmission ratio gear, a mechanism of linkages, and the supporting electronics. Its nonlinear transmission ratio changes smoothly from a minimum value at the middle position of the mobile link to an ad-infinitum value at the extreme positions of the mobile link. Nevertheless, this drive has the additional advantage of presenting two possible magnitudes of the reduction ratio for each position of the mobile link, due to the particular configuration of the mechanism of linkages. This dual property permits the linkage mechanism to be arranged within the limits of one angle when the load (or external force) is small (for high displacement speeds), or within the limits of another angle when the load is greater (at accordingly smaller displacement velocities). Consequently, the Dual Smart Drive can be especially useful for climbing robots, which experience severe changes in external forces due to gravity while ascending or descending a vertical wall or an inclined surface. Moreover, use of this drive allows the motion speed of walking machines to be increased, because the Dual Smart Drive provides fast acceleration and deceleration at the beginning and at the end of the driving trajectory, for both legs and body (high absolute magnitude of the reduction ratio), while maintaining high speeds in the intermediate part of the driving trajectory for both legs and body (low absolute magnitude of the reduction ratio), and providing higher speed and lower torque for the motion of the legs and higher torque and lower speed for the movement of the body.

Using this kind of nonlinear actuator makes control systems more complex. A first approach for the Dual Smart Drive control was presented in (Fernández et al., 2003). That algorithm (originally created for resonance drives (Akinfiev, 1990)) divided the movement trajectory in each working regime into two equal parts, passive and active. In the passive part, the system operated under open-loop control while the angular positions and the angular velocities were recorded. In the active part, the algorithm mirrored the stored pairs of data and used them as the reference signal in phase-plane control. Thus, the system's behavior was perfectly symmetrical and took into account the intrinsic dynamics of the Dual Smart Drive. Nevertheless, any noise or disturbances during the passive part could have an unpredictable effect on the resulting scheme. To solve that problem, a combined backstepping/time-optimal control strategy is proposed, which increases robustness and guarantees asymptotic tracking. The basic idea is to use the backstepping design technique to develop Lyapunov-based nonlinear controllers for the Dual Smart Drive that conduct asymptotic tracking of the reference trajectories, which have 
been suitably selected using the time-optimal control method (Athans and Falb, 1966). The problem is also extended to include bounded control inputs (Lin and Sontag, 1991). The backstepping approach (Kanellakopoulos et al., 1991), (Kokotovic, 1992) is a flexible, powerful, well-studied (Isidori, 1989), (Khalil, 2002) design tool for stabilizing nonlinear systems in output feedback and strict feedback forms, for both tracking and regulation purposes (Krstic et al., 1995). The key idea of this technique is the systematic construction of a Lyapunov function for the closed loop, which allows its stability properties to be analyzed. Thus, at every step of backstepping, a new Control Lyapunov Function (CLF) is constructed by augmentation of the CLF from the previous step by a term that penalizes the error between a "virtual control" and its desired value (the so-called "stabilizing function"). Thus, the derivative of the Lyapunov function can be made negative definite by a variety of control laws, rather than by a specific control law.

The rest of the paper is organized as follows. Section 2 describes the Dual Smart Drive and its nonlinear mathematical model. Section 3 is devoted to the time-optimal control problem for the calculation of the reference trajectories. Section 4 explains the nonlinear controller design using the backstepping technique. Section 5 discusses and solves the problem of stabilization with bounded control. Section 6 demonstrates through simulations and experimental testing not only the improved performance of the Dual Smart Drive in comparison with other actuators, but also the effectiveness and feasibility of the proposed nonlinear control algorithms. Lastly, section 7 summarizes the major conclusions and future research directions.

\section{System Description}

The design of the actuator was presented in (Fernández et al., 2003). It consists of a crank connected to the reducing gear of a DC motor, a mobile link that rotates around a fixed point, and a slider that slips along the mobile link in a radial direction thanks to the movement of the crank to which it is connected. An essential characteristic of this actuator is that the length of the crank is smaller than the distance between the mobile link's rotation axis and the crank's rotation axis (see Figure 2). The kinematics is determined by the following parameters: $\ell_{M L}=$ distance between the mobile link's rotation axis and the crank's rotation axis; $\ell_{C}=$ length of the crank; $\varphi=$ angular position of the rotor measured clockwise from the $o x$ axis; $\alpha=$ angular position of the crank measured clockwise from the $o x$ axis; and $\beta=$

nRF-JAR-04-034.tex; 28/07/2005; 18:32; p.4 

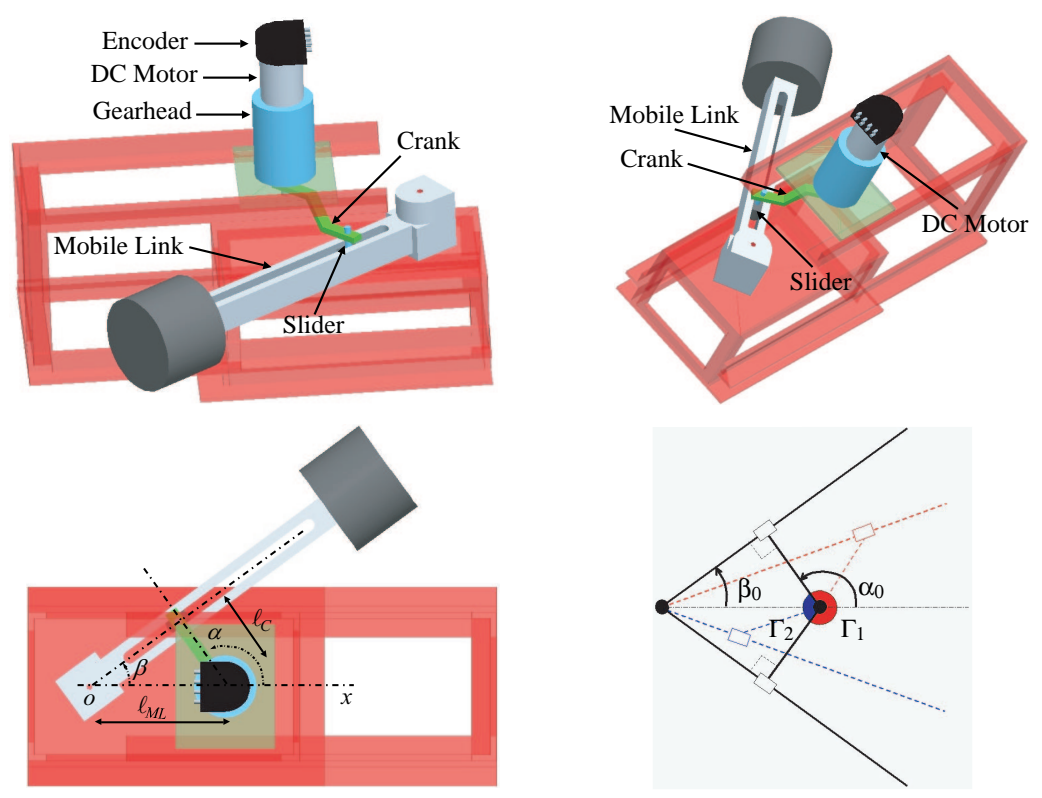

Figure 2. Elements and kinematic schema of the Dual Smart Drive.

angular position of the mobile link measured clockwise from the $o x$ axis. Taking into account that $\ell_{C}<\ell_{M L}$, the mobile link will move between two extreme positions (see Figure 2):

$$
-\beta_{0}<\beta<\beta_{0} \quad \text { where } \beta_{0}=\arcsin \left(\frac{\ell_{C}}{\ell_{M L}}\right) .
$$

The mobile link can be shifted from one end position to the other in two different ways: by displacement of the crank within the limits of angle $\Gamma_{1}$ (so-called first regime) or by displacement of the crank within the limits of angle $\Gamma_{2}$ (so-called second regime). The variation of the angular position of the mobile link, $\beta$, as a function of the angular position of the crank, $\alpha$, is given by:

$$
\beta=\operatorname{arctg}\left(\frac{\operatorname{Sin} \alpha}{\left(\ell_{M L} / \ell_{C}\right)+\operatorname{Cos} \alpha}\right) .
$$

Since the relationship between the angular position of the rotor, $\varphi$, and the angular position of the crank is $\varphi=K_{G} \alpha,\left(K_{G}=\right.$ constant transmission of the reduction gear), the relationship can be rewritten as:

$$
\beta=\operatorname{arctg}\left(\frac{\operatorname{Sin}\left(\varphi / K_{G}\right)}{\left(\ell_{M L} / \ell_{C}\right)+\operatorname{Cos}\left(\varphi / K_{G}\right)}\right)
$$


The transmission ratio between the reducing gear and the mobile link can be calculated using:

$$
K_{D}=\frac{\dot{\alpha}}{\dot{\beta}},
$$

where $\dot{\alpha}$ is the angular velocity of the crank and $\dot{\beta}$ is the angular velocity of the mobile link. As a result,

$$
K_{D}=\frac{1+\left(\ell_{M L} / \ell_{C}\right)^{2}+2\left(\ell_{M L} / \ell_{C}\right) \operatorname{Cos} \alpha}{1+\left(\ell_{M L} / \ell_{C}\right) \operatorname{Cos} \alpha} .
$$

Then, the nonlinear transmission ratio, as a function of the angular position of the rotor, is given by:

$$
K_{D(\varphi)}=\frac{1+\left(\ell_{M L} / \ell_{C}\right)^{2}+2\left(\ell_{M L} / \ell_{C}\right) \operatorname{Cos}\left(\varphi / K_{G}\right)}{1+\left(\ell_{M L} / \ell_{C}\right) \operatorname{Cos}\left(\varphi / K_{G}\right)} .
$$

The angular velocity of the rotor as a function of the angular velocity of the mobile link is given by:

$$
\dot{\varphi}=K_{G} K_{D(\varphi)} \dot{\beta} .
$$

Consequently, the angular acceleration of the mobile link may be stated as:

$$
\ddot{\beta}=\frac{\ddot{\varphi}}{K_{G} K_{D(\varphi)}}-\frac{\dot{K}_{D(\varphi)} \dot{\varphi}}{K_{G} K_{D(\varphi)}^{2}} .
$$

Equation (5) is a $2 \pi$ periodic equation, consisting in two different parts: one with negative values (part $\Gamma_{2}$ ), and one with positive values (part $\Gamma_{1}$ ) (see Figure 3(a)). The negative magnitude of the reduction ratio means that the crank and the mobile link are rotating in opposite directions. It is interesting to note that the reduction ratio tends to infinity at the end points, $-\beta_{0}, \beta_{0}$, where the crank is perpendicular to the mobile link. At these points, the deviation of the mobile link from its medium position is maximal. That is why the movement from one end position to the other ensures the best change of the reduction ratio for maintaining high accelerations of the mobile link at the beginning and at the end of driving (high absolute magnitude of the reduction ratio) and for maintaining high speeds in the intermediate part of the trajectory (low absolute magnitude of the reduction ratio). Moreover, when the mobile link is moved by displacement of the crank within the limits of angle $\Gamma_{1}$, the absolute average magnitude of the reduction ratio will be greater than when displacement is within the limits of angle $\Gamma_{2}$ (see Figure $3(\mathrm{~b})$ ). The movement of the crank can therefore be used within the limits of angle $\Gamma_{2}$ when the load is small (i.e. for

nRF-JAR-04-034.tex; 28/07/2005; 18:32; p.6 
moving the robot's leg) and displacement speeds are high, or within the limits of angle $\Gamma_{1}$ when the load is great (i.e. for moving the robot's body), at correspondingly smaller displacement velocities. Therefore, the drive allows the mobile link to shift easily from one end position to another, with two different laws for changing the drive's reduction ratio (Fernández et al., 2003), and makes independent tuning for two different movements possible. One important point is that, although the mobile link of the Dual Smart Drive could move from any position to any other position between $-\beta_{0}$ and $\beta_{0}$, only movement from one end position to the other ensures maximum effectiveness.

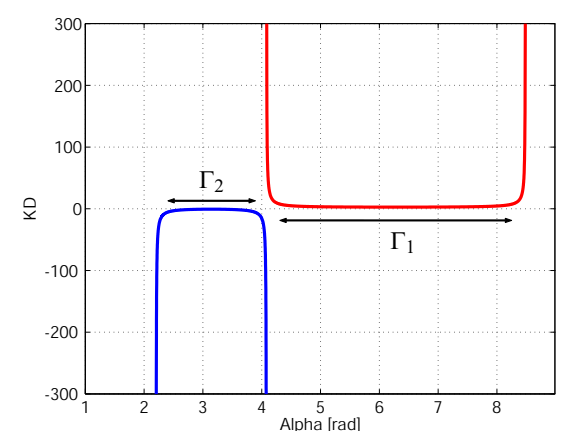

(a)

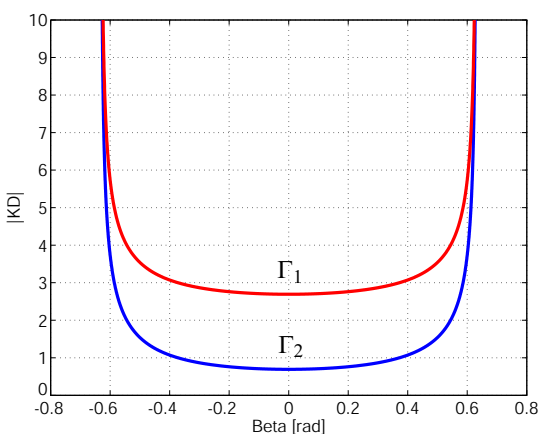

(b)

Figure 3. (a) $K_{D}$ vs. $\alpha$. (b) Absolute magnitudes of $K_{D}$ vs. $\beta$.

Figures 4 and 5 display some examples of Dual Smart Drive connections with legged robots. In Figure 4, the Dual Smart Drive is used to perform a horizontal movement of the legs and the body in a multi-legged robot with Cartesian DOF. In this configuration, gravity does not affect the drive's dynamics. Additionally, this design facilitates analysis of the drive's dynamics within the whole robot system, without having to take into consideration the kinematic connection of leg parts (unlike designs where the robot has rotational DOF). There is only one problem: synchronization of leg velocities when all the legs rest on the horizontal plane and all the Dual Smart Drives are working simultaneously to move the robot's body horizontally. Accordingly, it is necessary to have a control system that can provide high-quality tracking of the reference trajectory for the Dual Smart Drive, which is characterized by several nonlinearities. A control of this kind comes under consideration in sections 4,5 and 6 of this paper. Although the Dual Smart Drive could also be used for rotational or progressive movement with vertical displacement of the leg's center of gravity, as 
shown in Figure 5, the analysis of this configuration lies beyond the scope of this paper.

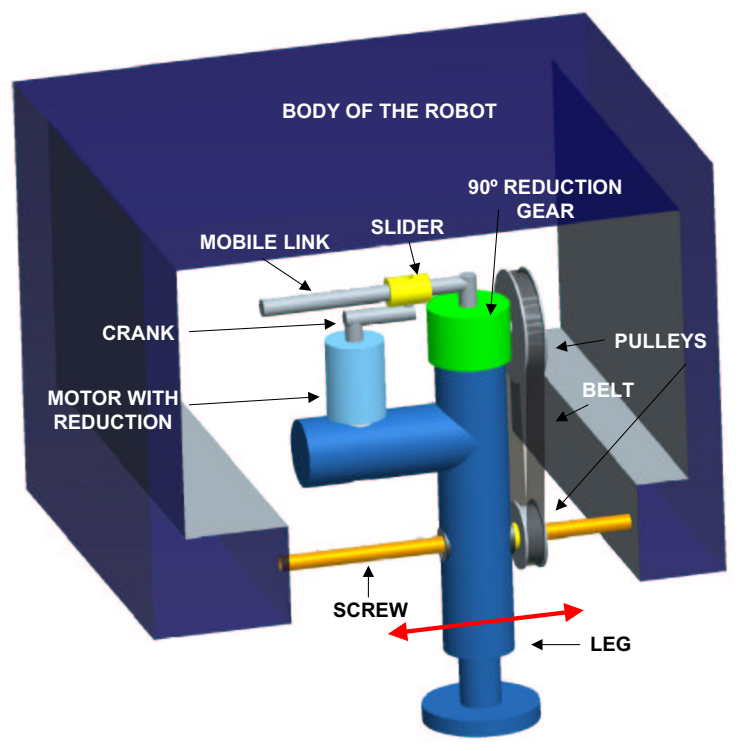

Figure 4. Example of a Dual Smart Drive connection on a legged robot.

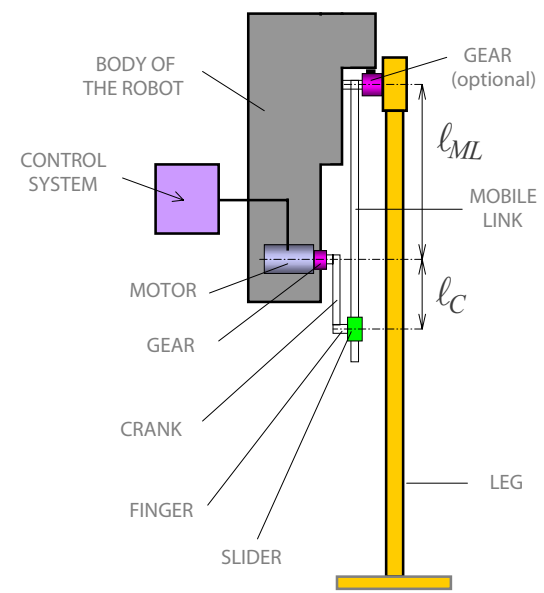

Figure 5. Example of a vertical Dual Smart Drive connection on a legged robot.

Resuming the analysis of the Dual Smart Drive, note that, at the singular points where the drive changes its working regime, the velocities are null. This essential feature enables the Dual Smart Drive's 
dynamics to be modeled independently for each working regime and allows the two models to be combined for an overall representation. The drive is also assumed to be operating horizontally in order to achieve gravitational decoupling. Bearing these facts in mind, the mobile link equation is given by:

$$
J_{i} \ddot{\beta}_{i}=K_{D} K_{G} M_{i}-b_{i} \dot{\beta}_{i}-M_{F R i} \operatorname{sign}\left(\dot{\beta}_{i}\right),
$$

where $J_{i}$ is the equivalent inertia of the mobile link in each working regime $i=1,2, \ddot{\beta}_{i}$ is the angular acceleration of the mobile link, $M_{i}$ is the moment that acts on the mobile link, $b_{i}$ is the equivalent viscosity friction coefficient, and $M_{F R i}$ is the moment of dry friction during the movement in each regime. The rotor equation is given by:

$$
J_{M} \ddot{\varphi}_{i}=\tau_{M i}-M_{i}-b_{M} \dot{\varphi}_{i},
$$

where $J_{M}$ is the rotor inertia, $\ddot{\varphi}_{i}$ is the angular acceleration of the rotor, $\tau_{M i}$ is the motor torque, $M_{i}$ is the moment that acts on the rotor gear, and $b_{M}$ is the viscosity friction coefficient on the motor shaft.

The motor torque $\tau_{M i}$ is given by:

$$
\tau_{M i}=k_{m} I_{A i},
$$

where $k_{m}$ is the torque constant and $I_{A i}$ is the armature current:

$$
I_{A i}=\frac{1}{R_{M}}\left[u_{i}-k_{E} \dot{\varphi}_{i}\right],
$$

where $u_{i}$ is the voltage fed into motor by control system, $R_{M}$ is the motor resistance, and $k_{E}$ is the back-EMF constant.

Combining all these equations, the dynamic model of the system with a nonlinear transmission ratio is:

$$
\begin{aligned}
& \dot{x}_{1 i}= x_{2 i}, \\
& \dot{x}_{2 i}=\frac{1}{J_{M}+\frac{J_{G i}}{K_{D\left(x_{1 i}\right)}^{2}}}\left[\left(\frac{J_{G i} \dot{K}_{D\left(x_{1 i}, x_{2 i}\right)}}{K_{D\left(x_{1 i}\right)}^{3}}-K_{M a}-\right.\right. \\
&\left.\left.-\frac{b_{G i}}{K_{D\left(x_{1 i}\right)}^{2}}\right) x_{2 i}-\frac{M_{F R G i}}{K_{D\left(x_{1 i}\right)}} \operatorname{sign}\left(\frac{x_{2 i}}{K_{D\left(x_{1 i}\right)} K_{G}}\right)\right]+ \\
&+\frac{K_{M b}}{J_{M}+\frac{J_{G i}}{K_{D\left(x_{1 i}\right)}^{2}}} u_{i} .
\end{aligned}
$$


where $x_{1 i}$ denotes the angular position of the rotor, $\varphi ; x_{2 i}$, the corresponding angular velocity, $\dot{\varphi} ; \dot{K}_{D\left(\varphi_{i}\right)}$, the derivative of the nonlinear transmission ratio given by:

$$
\dot{K}_{D\left(\varphi_{i}\right)}=\frac{\frac{\ell_{M L} \dot{\varphi}_{i}}{\ell_{C} K_{G}} \sin \left(\frac{\varphi_{i}}{K_{G}}\right)\left[\left(\frac{\ell_{M L}}{\ell_{C}}\right)^{2}-1\right]}{\left[1+\frac{\ell_{M L}}{\ell_{C}} \cos \left(\frac{\varphi_{i}}{K_{G}}\right)\right]^{2}}
$$

and

$$
\begin{gathered}
J_{G i}=\frac{J_{i}}{K_{G}^{2}}, \quad K_{M a}=b_{M}+\frac{k_{E} k_{m}}{R_{M}}, \quad b_{G i}=\frac{b_{i}}{K_{G}^{2}}, \\
M_{F R G i}=\frac{M_{F R i}}{K_{G}}, \quad K_{M b}=\frac{k_{m}}{R_{M}} .
\end{gathered}
$$

\section{Time-Optimal Control}

The section above argues that the movement from one end position to the other ensures a favorable change of the reduction ratio for each working regime. The desired control objective is, then, to make this displacement in a minimum time using all the capabilities that the electromotor and the transmission have available. For this reason, a time-optimal control (Athans and Falb, 1966) is used to calculate the reference trajectories.

For nonlinear systems, optimal control theory only provides the necessary conditions for optimality. Hence, only a set of candidate controls can be deduced using the general theory. So, once the equations of motion have been derived, Pontryagin's Minimum Principle is applied to obtain the necessary conditions for optimality. Then, the equations for the state and costate vector that satisfy the necessary conditions are determined, and, subsequently, the control sequences that can be candidates for time-optimal control are obtained. The control problem is to minimize the cost functional

$$
\Psi\left(u_{i}\right)=\int_{t_{0}}^{T} d t=T-t_{0}, \quad T \text { is free, }
$$

subject to a magnitude-input constraint of the form $\left|u_{i}(t)\right| \leq u_{\max }$, $\forall t \in\left[t_{0}, T\right]$. The Hamiltonian function for system (13) and cost functional (16) is given by:

$$
H_{i}(x, p, u)=1+x_{2 i} p_{1 i}+f\left(x_{1 i}, x_{2 i}\right) p_{2 i}+K\left(x_{1 i}\right) u_{i} p_{2 i},
$$

nRF-JAR-04-034.tex; 28/07/2005; 18:32; p.10 
where,

$$
\begin{aligned}
f\left(x_{1 i}, x_{2 i}\right)= & \frac{1}{J_{M}+\frac{J_{G i}}{K_{D\left(x_{1}\right)}^{2}}}\left[\left(\frac{J_{G i} \dot{K}_{D\left(x_{1}, x_{2}\right)}}{K_{D\left(x_{1}\right)}^{3}}-K_{M a}-\right.\right. \\
& \left.\left.-\frac{b_{G i}}{K_{D\left(x_{1}\right)}^{2}}\right) x_{2 i}-\frac{M_{F R G i}}{K_{D\left(x_{1}\right)}} \operatorname{sign}\left(\frac{x_{2 i}}{K_{D\left(x_{1}\right)} K_{G}}\right)\right],
\end{aligned}
$$

and

$$
K\left(x_{1 i}\right)=\frac{k_{m}}{R_{M}\left(J_{M}+\frac{J_{i}}{K_{D\left(x_{1}\right)}^{2} K_{G}^{2}}\right)} .
$$

Since the Hamiltonian function is linear at $u_{i}$, the optimal control is of the form

$$
u_{i}^{*}(t)=u_{\max } \operatorname{sign}\left[K\left(x_{1 i}^{*}(t)\right) p_{2 i}^{*}(t)\right]
$$

almost everywhere at $\left[t_{0}, T^{*}\right]$, where $T^{*}$ is the minimum time, and $x_{1 i}^{*}(t)$ and $p_{2 i}^{*}(t)$ are the state and costate trajectories under the optimal control law. Thus, the time-optimal control is bang-bang. This means that the state space can be partitioned into two regions, one in which $u_{i}=u_{\max }$ and another in which $u_{i}=-u_{\max }$. The boundary between the two regions is called the switching curve. For second-order systems such as this one, the switching curve can be determined by plotting system trajectories in the phase plane for the two extreme control values. Figure 6 shows trajectories of system (13) for $u_{i}=u_{\max }$ (solid curves) and $u_{i}=-u_{\max }$ (dashed curves). The arrows indicate the direction of motion of the state. All the trajectories due to $u_{i}=$ $+u_{\text {max }}$ can be seen to tend to the line $x_{2 i}=c_{1}$, and all the trajectories due to $u_{i}=-u_{\max }$ can be seen to tend to the line $x_{2 i}=-c_{1}$. The trajectories that pass through the origin are labeled $\gamma_{+}$and $\gamma_{-}$(Athans and Falb, 1966). The $\gamma_{+}$curve is the locus of all points $\left(x_{1 i}, x_{2 i}\right)$ that can be forced to $(0,0)$ by the control $u_{i}=+u_{\max }$, and the $\gamma_{-}$curve is the locus of all points $\left(x_{1 i}, x_{2 i}\right)$ that can be forced to $(0,0)$ by the control $u_{i}=-u_{\max }$. The $\gamma$ curve, called the switching curve, is the union of the $\gamma_{+}$and $\gamma_{-}$curves, and it divides the state plane into two regions, $R_{+}$and $R_{-}$. $R_{+}$consists of the points to the left of the $\gamma$ switching curve, and $R_{-}$consists of the points to the right of the $\gamma$ switching curve (cf. Figure 7). Since the bang-bang control has a finite number of switches in every bounded time interval, it can be demonstrated that the extremal controls for system (13) can 
switch at most once, and that only the four control sequences $\left\{+u_{\max }\right\}$, $\left\{-u_{\max }\right\},\left\{+u_{\max },-u_{\max }\right\}$, and $\left\{-u_{\max },+u_{\max }\right\}$ can be candidates for time-optimal control. The arguments are illustrated in Figure 8. If the initial state $\Xi=\left(\xi_{1}, \xi_{2}\right)$ belongs to the $\gamma_{+}$, by definition, control sequence $\left\{+u_{\max }\right\}$ results in trajectory $\Xi 0$, which reaches the origin. Control sequence $\left\{-u_{\max }\right\}$ results in trajectory $\Xi A^{\prime}$, which never reaches the origin. Control sequence $\left\{+u_{\max },-u_{\max }\right\}$ results in trajectories of the $\Xi B^{\prime} C^{\prime}$ type, which never reach the origin. Control sequence $\left\{-u_{\max },+u_{\max }\right\}$ results in trajectories of the $\Xi D^{\prime} E^{\prime}$ type, which never reach the origin.

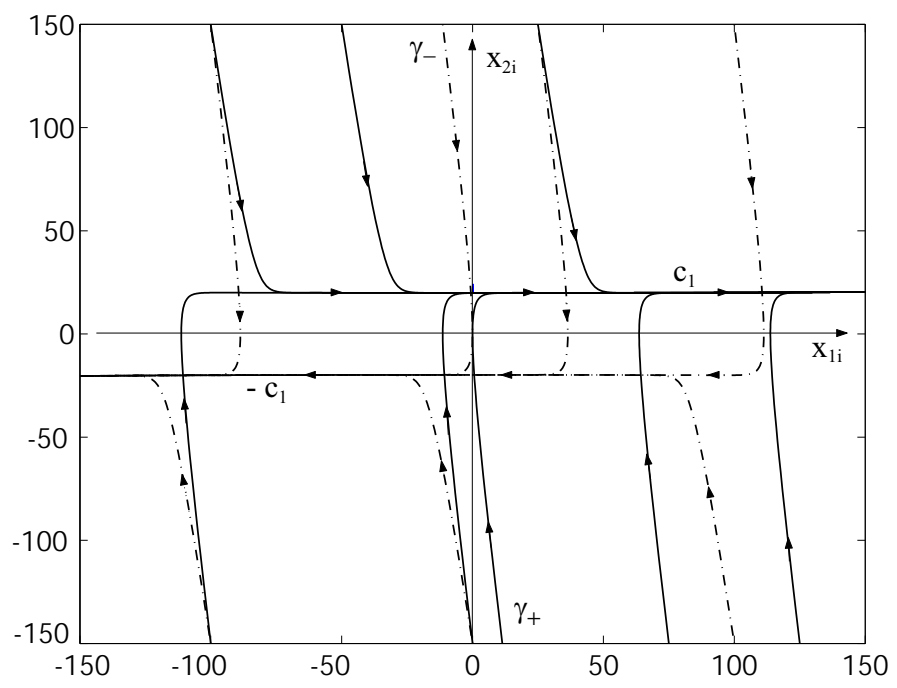

Figure 6. State-plane trajectories for the system given by (13). For illustration purposes, $u_{\max }=1$.

Therefore, if the initial state is on the $\gamma_{+}$curve, then, of all the control sequences that are candidates for minimum-time control, only $\left\{+u_{\max }\right\}$ can force state $\Xi$ to 0 . Thus, by elimination, it must be time optimal. Using analogous arguments, it can be shown that if the initial state belongs to the $\gamma_{-}$curve, then the time-optimal control is $u_{i}=-u_{\max }$. Thus, the time-optimal control law for initial states on the $\gamma$ curve has been derived. Let us now consider an initial state $X$ that belongs to the $R_{+}$region. If the $\left\{+u_{\max }\right\}$ control sequence is used, the resulting trajectory is $X F^{\prime}$, shown in Figure 8 , which never reaches the origin. If the $\left\{-u_{\max }\right\}$ sequence is applied, the resulting trajectory, $X G^{\prime}$, never reaches the origin. If the $\left\{-u_{\max },+u_{\max }\right\}$ sequence is applied, the resulting trajectory is of the $X H^{\prime} I^{\prime}$ type, which does not reach the origin. However, if 


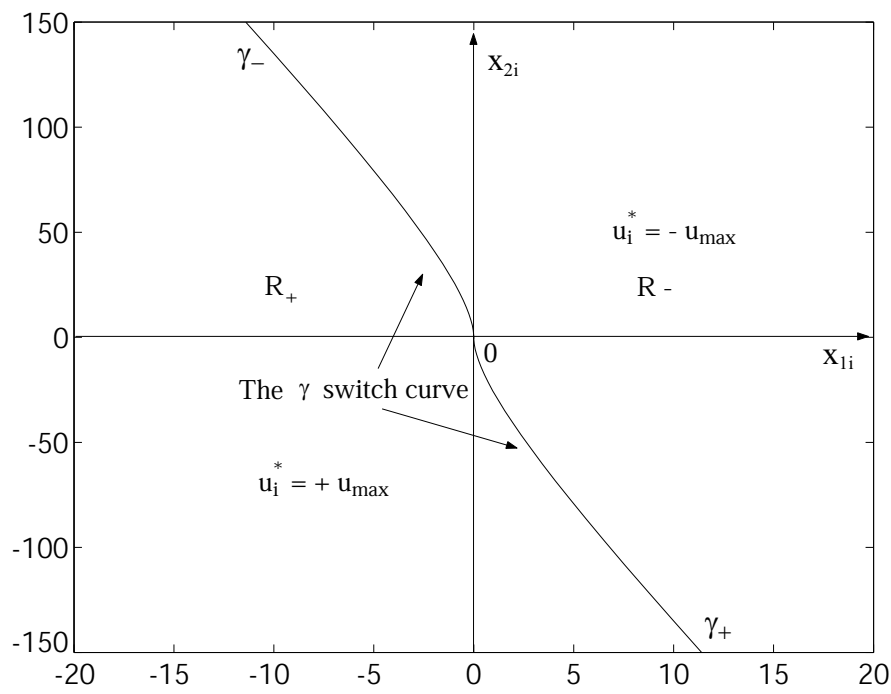

Figure 7. Switching curve for second-order nonlinear system (13). For illustration purposes, $u_{\max }=1$.

the $\left\{+u_{\max },-u_{\max }\right\}$ sequence is used, then the origin can be reached along the $X J^{\prime} 0$ trajectory, provided that the transition from the $u_{i}=$ $+u_{\max }$ control to $u_{i}=-u_{\max }$ occurs at point $J^{\prime}$, that is, at the precise moment that the trajectory crosses the $\gamma$ switching curve. This is true for every state in $R_{+}$. Thus, by the process of elimination, the conclusion is reached that the $\left\{+u_{\max },-u_{\max }\right\}$ sequence is time optimal for every state in $R_{+}$, provided that the control switches from $u_{i}=+u_{\max }$ to $u_{i}=-u_{\max }$ at the $\gamma$ switching curve. Using identical arguments, it may be concluded that, when the initial state belongs to $R_{-}$, the $\left\{-u_{\max },+u_{\max }\right\}$ sequence is time optimal with the transition from $-u_{\max }$ to $u_{\max }$ over $\gamma$. The $u_{i}^{*}$ time-optimal control can therefore be written as a function of the state as follows:

$$
\begin{array}{ll}
u_{i}^{*}=u_{i}^{*}\left(x_{1 i}, x_{2 i}\right)=+u_{\max } & \text { for all }\left(x_{1 i}, x_{2 i}\right) \in \gamma_{+} \cup R_{+}, \\
u_{i}^{*}=u_{i}^{*}\left(x_{1 i}, x_{2 i}\right)=-u_{\max } & \text { for all }\left(x_{1 i}, x_{2 i}\right) \in \gamma_{-} \cup R_{-} .
\end{array}
$$

Bang-bang control is useful for establishing a theoretical bound on the best possible controlled system performance, but it is generally quite difficult to apply to practical problems (Song and Smith, 2000), (Meckl and Seering, 1985). Usually, its performance degrades severely with modeling inaccuracies, unpredicted external disturbances or measurement noise. A combination of time-optimal control and 
backstepping is proposed to add stability by using the time-optimal trajectories as reference values for a controller designed using integrator backstepping. The problem is also discussed for bounded control inputs in section 5 . Thus, the approaches will be quasi-time optimal rather than exactly time optimal.

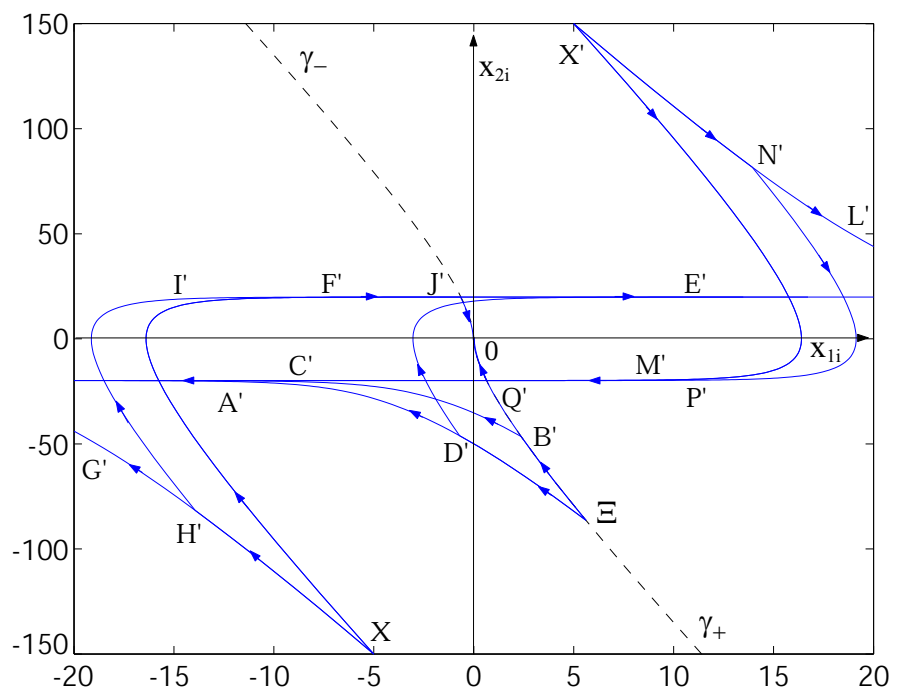

Figure 8. Various trajectories generated by the four possible control sequences. For illustration purposes, $u_{\max }=1$.

\section{Backstepping}

In order to solve the tracking problem, a nonlinear trajectory-tracking controller is proposed following the integrator backstepping technique (Kanellakopoulos et al., 1991), (Khalil, 2002), (Kokotovic, 1992), (Krstic et al., 1995). Firstly, a coordinate transformation is introduced 
for system (13):

$$
\begin{aligned}
& \dot{e}_{1 i}=e_{2 i}+x_{d 2 i}-\dot{x}_{d 1 i}, \\
& \dot{e}_{2 i}=\frac{1}{J_{M}+\frac{J_{G i}}{K_{D\left(e_{1 i}+x_{d 1 i}\right)}^{2}}}\left[\left(-K_{M a}-\frac{b_{G i}}{K_{D\left(e_{1 i}+x_{d 1 i}\right)}^{2}}+\right.\right. \\
& \left.+\frac{J_{G i} \dot{K}_{D\left(e_{1 i}+x_{d 1 i}, e_{2 i}+x_{d 2 i}\right)}}{K_{D\left(e_{1 i}+x_{d 1 i}\right)}^{3}}\right)\left(e_{2 i}+x_{d 2 i}\right)- \\
& \left.-\frac{M_{F R G i}}{K_{D\left(e_{1 i}+x_{d 1 i}\right)}} \operatorname{sign}\left(\frac{e_{2 i}+x_{d 2 i}}{K_{D\left(e_{1 i}+x_{d 1 i}\right)} K_{G}}\right)\right]+ \\
& +\frac{K_{M b}}{J_{M}+\frac{J_{G i}}{K_{D\left(e_{1 i}+x_{d 1 i}\right)}^{2}}} u_{i}-\dot{x}_{d 2 i} .
\end{aligned}
$$

where $e_{1 i}=x_{1 i}-x_{d 1 i}, e_{2 i}=x_{2 i}-x_{d 2 i}$ denote the position and velocity tracking errors, and $x_{d 1 i}=x_{1 i}^{*}$ and $x_{d 2 i}=x_{2 i}^{*}$, for $i=1,2$, denote the time-optimal trajectories determined in Section 3. Now, a smooth positive definite Lyapunov-like function is defined as follows:

$$
V_{1 i}=\frac{1}{2} e_{1 i}^{2}
$$

Its derivative is given by:

$$
\dot{V}_{1 i}=e_{1 i}\left(e_{2 i}+x_{d 2 i}-\dot{x}_{d 1 i}\right) \text {. }
$$

Next, $e_{2 i}$ is regarded as a virtual control law to make $\dot{V}_{1 i}$ negative. This is achieved by setting $e_{2 i}$ equal to $-x_{d 2 i}+\dot{x}_{d 1 i}-k_{1 i} e_{1 i}$, for some positive constant $k_{1 i}$. To accomplish this, an error variable $z_{2 i}$ that we would like to set to zero is introduced:

$$
z_{2 i}=e_{2 i}+x_{d 2 i}-\dot{x}_{d 1 i}+k_{1 i} e_{1 i} .
$$

Then $\dot{V}_{1 i}$ becomes:

$$
\dot{V}_{1 i}=z_{2 i} e_{1 i}-k_{1 i} e_{1 i}^{2} .
$$


To backstep, system (22) is transformed into the form:

$$
\begin{aligned}
& \dot{e}_{1 i}=-k_{1 i} e_{1 i}+z_{2 i} \\
& \dot{z}_{2 i}=\frac{1}{J_{M}+\frac{J_{G i}}{K_{D\left(e_{1 i}+x_{d 1 i}\right)}^{2}}}\left[\left(\frac{J_{G i} \dot{K}_{D\left(e_{1 i}+x_{d 1 i}, z_{2 i}+\dot{x}_{d 1 i}-k_{1 i} e_{1 i}\right)}}{K_{D\left(e_{1 i}+x_{d 1 i}\right)}^{3}}-\right.\right. \\
& \\
&\left.-K_{M a}-\frac{b_{G i}}{K_{D\left(e_{1 i}+x_{d 1 i}\right)}^{2}}\right)\left(z_{2 i}+\dot{x}_{d 1 i}-k_{1 i} e_{1 i}\right)- \\
&\left.\quad-\frac{M_{F R G i}}{K_{D\left(e_{1 i}+x_{d 1 i}\right)}} \operatorname{sign}\left(\frac{z_{2 i}+\dot{x}_{d 1 i}-k_{1 i} e_{1 i}}{K_{D\left(e_{1 i}+x_{d 1 i}\right)} K_{G}}\right)\right]+ \\
&+\frac{K_{M b}}{J_{M}+\frac{J_{G i}}{K_{D\left(e_{1 i}+x_{d 1 i}\right)}^{2}}} u_{i}-\ddot{x}_{d 1 i}+k_{1 i} \dot{e}_{1 i} .
\end{aligned}
$$

Now, a new control Lyapunov function, $V_{2 i}$, is built by augmenting the control Lyapunov function $V_{1 i}$ obtained in the previous step using a stabilization function. This function penalizes the error between the virtual control and its desired value. So, taking

$$
V_{2 i}=V_{1 i}+\frac{1}{2} \kappa_{i} z_{2 i}^{2}
$$


as a composite Lyapunov function, we obtain:

$$
\begin{gathered}
\dot{V}_{2 i}=-k_{1 i} e_{1 i}^{2}+\kappa_{i} z_{2 i}\left[\frac { 1 } { J _ { M } + \frac { J _ { G i } } { K _ { D ( e _ { 1 i } + x _ { d 1 i } ) } ^ { 2 } } } \left[\left(-\frac{b_{G i}}{K_{D\left(e_{1 i}+x_{d 1 i}\right)}^{2}}+\right.\right.\right. \\
+\frac{\left.J_{G i} \dot{K}_{D\left(e_{1 i}+x_{d 1 i}, z_{2 i}+\dot{x}_{d 1 i}-k_{1 i} e_{1 i}\right)}-K_{M a}^{3}\right)\left(z_{2 i}+\dot{x}_{d 1 i}\right.}{D\left(e_{1 i}+x_{d 1 i}\right)} \\
\left.\left.-k_{1 i} e_{1 i}\right)-\frac{M_{F R G i}}{K_{D\left(e_{1 i}+x_{d 1 i}\right)}} \operatorname{sign}\left(\frac{z_{2 i}+\dot{x}_{d 1 i}-k_{1 i} e_{1 i}}{K_{D\left(e_{1 i}+x_{d 1 i}\right)} K_{G}}\right)\right]+ \\
\left.+\frac{K_{M a}}{J_{M}+\frac{J_{G i}}{K_{D\left(e_{1 i}+x_{d 1 i}\right)}^{2}}} u_{i}+\frac{e_{1 i}}{\kappa_{i}}-\ddot{x}_{d 1 i}+k_{1 i} \dot{e}_{1 i}\right]
\end{gathered}
$$

Choosing

$$
\begin{aligned}
u_{i}= & \left(\frac{J_{M}}{K_{M b}}+\frac{J_{G i}}{K_{M b} K_{D\left(e_{1 i}+x_{d 1 i}\right)}^{2}}\right)\left[-\frac{e_{1 i}}{\kappa_{i}}+\ddot{x}_{d 1 i}-k_{1 i} \dot{e}_{1 i}\right. \\
& -\frac{1}{J_{M}+\frac{J_{G i}}{K_{D\left(e_{1 i}+x_{d 1 i}\right)}^{2}}\left[\left(\frac{J_{G i} \dot{K}_{D\left(e_{1 i}+x_{d 1 i}, z_{2 i}+\dot{x}_{d 1 i}-k_{1 i} e_{1 i}\right)}}{K_{D\left(e_{1 i}+x_{d 1 i}\right)}^{3}}\right.\right.} \\
& \left.-K_{M a}-\frac{b_{G i}}{K_{D\left(e_{1 i}+x_{d 1 i}\right)}^{2}}\right)\left(z_{2 i}+\dot{x}_{d 1 i}-k_{1 i} e_{1 i}\right)- \\
& \left.\left.-\frac{M_{F R G i}}{K_{D\left(e_{1 i}+x_{d 1 i}\right)}} \operatorname{sign}\left(\frac{z_{2 i}+\dot{x}_{d 1 i}-k_{1 i} e_{1 i}}{K_{D\left(e_{1 i}+x_{d 1 i}\right)} K_{G}}\right)\right]-k_{2 i} z_{2 i}\right]
\end{aligned}
$$

yields

$$
\dot{V}_{2 i}=-k_{1 i} e_{1 i}^{2}-\kappa_{i} k_{2 i} z_{2 i}^{2},
$$

where $k_{1 i}, k_{2 i}>0$. This implies asymptotical stability according to Lyapunov's stability theorem. 


\section{Boundedness of the Control Input}

Since the controller built with the backstepping technique can cause control-signal saturation in practical implementations, in this section the study is extended to include the case of a bounded input. Thus, a formula is used for a stabilizing feedback law with bounded control (Lin and Sontag, 1991), under the assumption that an appropriate control Lyapunov function is known. Consider transformed system (27), rewritten as:

$$
\dot{\eta}=: f_{i}(\eta)+g_{i}(\eta) u_{i} \quad \text { for } \quad \dot{\eta}=\left[\begin{array}{ll}
\dot{e}_{1 i} & \dot{z}_{2 i}
\end{array}\right]^{\mathrm{T}},
$$

where

$$
\begin{aligned}
& f_{i}(\eta):=\left[\begin{array}{ll}
f_{1 i} & f_{2 i}
\end{array}\right]^{\mathrm{T}}, \\
& f_{1 i}:=-k_{1 i} e_{1 i}+z_{2 i},
\end{aligned}
$$

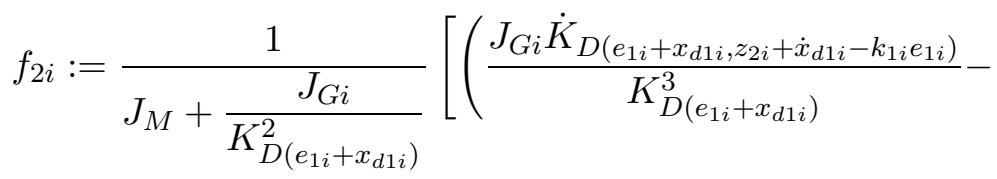

$$
\begin{aligned}
& \left.-K_{M a}-\frac{b_{G i}}{K_{D\left(e_{1 i}+x_{d 1 i}\right)}^{2}}\right)\left(z_{2 i}+\dot{x}_{d 1 i}-k_{1 i} e_{1 i}\right)- \\
& \left.-\frac{M_{F R G i}}{K_{D\left(e_{1 i}+x_{d 1 i}\right)}} \operatorname{sign}\left(\frac{z_{2 i}+\dot{x}_{d 1 i}-k_{1 i} e_{1 i}}{K_{D\left(e_{1 i}+x_{d 1 i}\right)} K_{G}}\right)\right]-\ddot{x}_{d 1 i}+k_{1 i} \dot{e}_{1 i} ; \\
& g_{i}(\eta):=\left[\begin{array}{ll}
0 & g_{2 i}
\end{array}\right]^{\mathrm{T}}, \\
& g_{2 i}:=\frac{k_{m}}{R_{M}\left(J_{M}+\frac{J_{i}}{K_{D\left(e_{1 i}+x_{d 1 i}\right)}^{2} K_{G}^{2}}\right)},
\end{aligned}
$$

and the known appropriate control Lyapunov function (28)

$$
V_{2 i}=\frac{1}{2} e_{1 i}^{2}+\frac{1}{2} \kappa_{i} z_{2 i}^{2} .
$$

For the particular case of scalar-valued controls, the resulting bounded feedback law is

$$
u_{i}=-\Upsilon_{i}\left(x_{i}, u_{\max }\right) u_{\max },
$$


where

$$
\Upsilon_{i}\left(x_{i}, u_{\max }\right)=\frac{L_{f} V_{2 i}+\sqrt{\left(L_{f} V_{2 i}\right)^{2}+\left(u_{\max }\left\|L_{g} V_{2 i}\right\|\right)^{4}}}{u_{\max } L_{g} V_{2 i}\left[1+\sqrt{1+\left(u_{\max }\left\|L_{g} V_{2 i}\right\|\right)^{2}}\right]} .
$$

$L_{f} V_{2 i}$ and $L_{g} V_{2 i}$, which are the Lie derivatives of (35) with respect to the vector fields defining the system, are given by:

$$
\begin{aligned}
& L_{f} V_{2 i}=e_{1 i}\left(-k_{1 i} e_{1 i}+z_{2 i}\right)+\frac{\kappa_{i} z_{2 i}}{J_{M}+\frac{J_{G}}{K_{D\left(e_{1 i}+x_{d 1 i}\right)}^{2}}}\left[\left(-K_{M a}+\right.\right. \\
& \left.+\frac{J_{G i} \dot{K}_{D\left(e_{1 i}+x_{d 1 i}, z_{2 i}+\dot{x}_{d 1 i}-k_{1 i} e_{1 i}\right)}}{K_{D\left(e_{1 i}+x_{d 1 i}\right)}^{3}}-\frac{b_{G i}}{K_{D\left(e_{1 i}+x_{d 1 i}\right)}^{2}}\right)\left(z_{2 i}+\right. \\
& \left.\left.+\dot{x}_{d 1 i}-k_{1 i} e_{1 i}\right)-\frac{M_{F R G i}}{K_{D\left(e_{1 i}+x_{d 1 i}\right)}} \operatorname{sign}\left(\frac{z_{2 i}+\dot{x}_{d 1 i}-k_{1 i} e_{1 i}}{K_{D\left(e_{1 i}+x_{d 1 i}\right)} K_{G}}\right)\right]- \\
& -\ddot{x}_{d 1 i} \kappa_{i} z_{2 i}+k_{1 i} \dot{e}_{1 i} \kappa_{i} z_{2 i} ; \\
& L_{g} V_{2 i}=\frac{k_{m} \kappa_{i} z_{2 i}}{R_{M}\left(J_{M}+\frac{J_{i}}{K_{D\left(e_{1 i}+x_{d 1 i}\right)}^{2} K_{G}^{2}}\right)} .
\end{aligned}
$$

Since the derivative of $V_{2 i}$ along the solutions of (32) is given by:

$$
\dot{V}_{2 i}=L_{f} V_{2 i}+L_{g} V_{2 i} u_{i},
$$

then, using equations (36) and (37), if $L_{f} V_{2 i}<0$, stability is guaranteed. Nevertheless, if $L_{f} V_{2 i}>0$, the condition of $\mathrm{u}_{i}^{2}>\left(\frac{L_{f} V_{2 i}}{L_{g} V_{2 i}}\right)^{2}$ has to be satisfied to guarantee stability.

\section{Simulations and Experiments}

\subsection{Comparison of the Dual Smart Drive Performance With That of Other Actuators}

Several comparisons have been carried out through simulations and experimental tests to confirm that the Dual Smart Drive outperforms 
Table I. Quantitative performance comparison between classical drive and Dual Smart Drive.

\begin{tabular}{|c|c|c|}
\hline \multicolumn{2}{|c|}{ FIRST REGIME: MOVEMENT OF THE BODY } \\
\hline & CLASSICAL DRIVE & DUAL DRIVE \\
\hline VOLTAGE [V] & 4.8 & 4.8 \\
\hline TIME [S] & 2.41 & 0.75 \\
\hline ENERGY [J] & 28.52 & 7.85 \\
\hline
\end{tabular}

\begin{tabular}{|c|c|c|}
\hline \multicolumn{3}{|c|}{ SECOND REGIME: MOVEMENT OF THE LEG } \\
\hline & CLASSICAL DRIVE & DUAL DRIVE \\
\hline VOLTAGE [V] & 4.8 & 4.8 \\
\hline TIME [S] & 0.58 & 0.35 \\
\hline ENERGY [J] & 6.77 & 3.98 \\
\hline
\end{tabular}

other drives. Firstly, the performance of the Dual Smart Drive was experimentally compared with that of a classical drive consisting in a DC motor. For this test, a special prototype was used that is dynamically equivalent to the one presented in Figure 4 and can be connected to the Dual Smart Drive or a traditional DC motor. The Dual Smart Drive, using the same motor, and an arrangement with $\ell_{M L}=0.11 \mathrm{~m}$ and $\ell_{C}=0.065 \mathrm{~m}$, proved to reduce motion time by $68.74 \%$ and energy consumption by $72.45 \%$ during the first regime, and to reduce motion time by $39.63 \%$ and energy consumption by $41.15 \%$ during the second regime. Figures 9 (a) and 9(b) show, respectively, the phase plane and the energy consumption of both the Dual Smart Drive (solid lines) and the classical drive (dashed lines) during the movement of the body with bang-bang control. Figures 9(c) and 9(d) display, respectively, the phase plane and the energy consumption of both the Dual Smart Drive (solid lines) and the classical drive (dashed lines) during the movement of one leg with bang-bang control. Lastly, Table I summarizes the quantitative experimental results. 


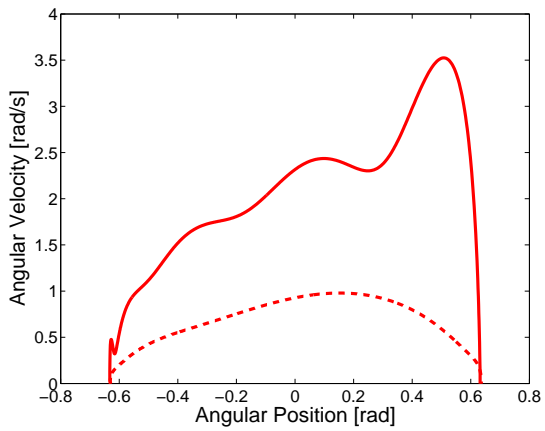

(a)

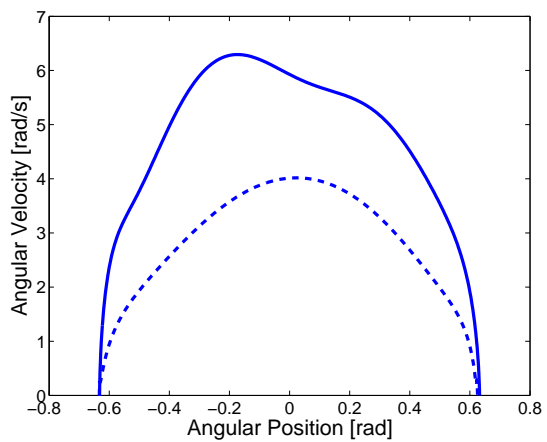

(c)

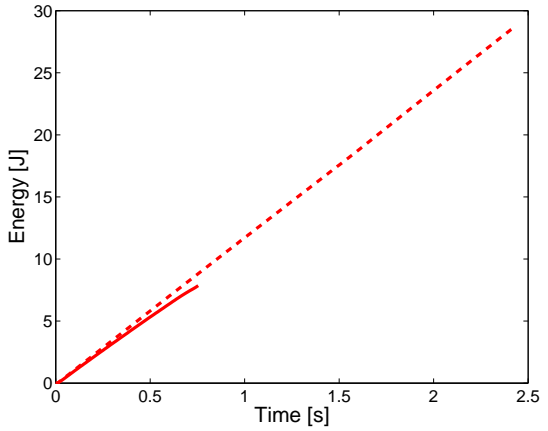

(b)

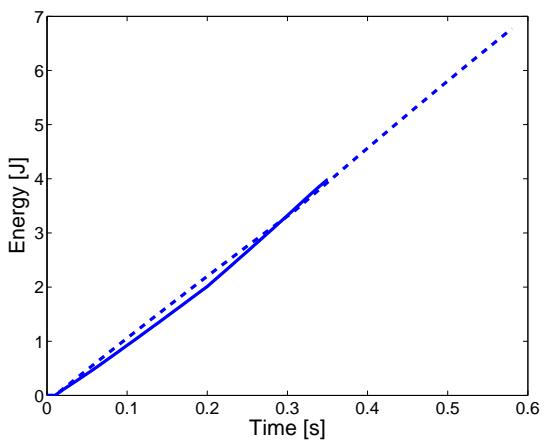

(d)

Figure 9. Performance comparison between classical drive (dashed lines) and Dual Smart Drive (solid lines).

Secondly, taking as an example the inertias that support one of the first joints of the four-legged robot Silo-4 (García and González de Santos, 2001) (see Figure 1(a)), the Dual Smart Drive's behavior was simulated in this joint using the same motor and the same reduction gear that the Silo-4 robot uses. The Dual Smart Drive proved to reduce motion time by a factor of 3.77 and energy consumption by a factor of 1.69 during the first regime, and to reduce motion time by a factor of 5.3 and energy consumption by a factor of 1.47 during the second regime. Figures 10(a) and 10(b) illustrate, respectively, the phase plane and the energy consumption of both the Dual Smart Drive (solid lines) and the first joint of the Silo-4 (dashed lines) during the movement of the robot's body. Figures 10(c) and 10(d) show, respectively, the phase plane and the energy consumption of both the Dual Smart Drive (solid lines) and the first joint of the Silo-4 (dashed lines) during the movement of one robot's leg. Table II summarizes the quantitative simulated results. 


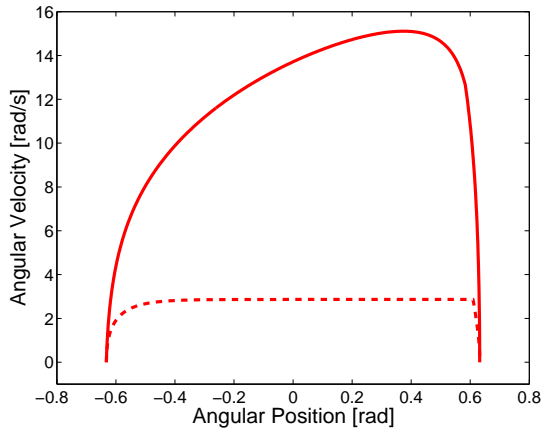

(a)

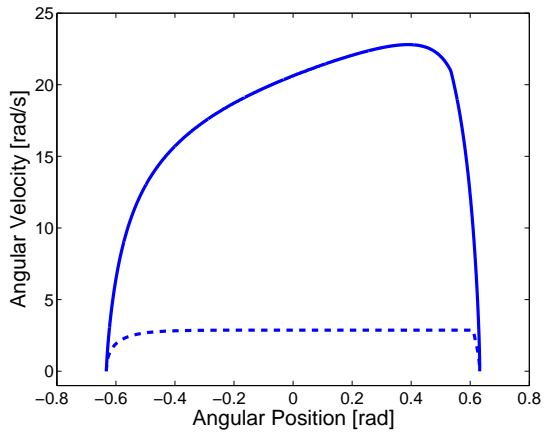

(c)

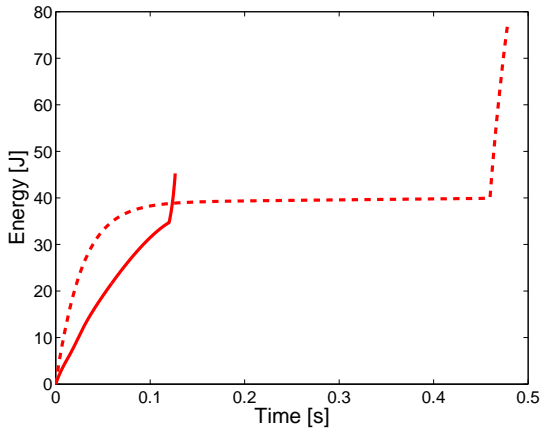

(b)

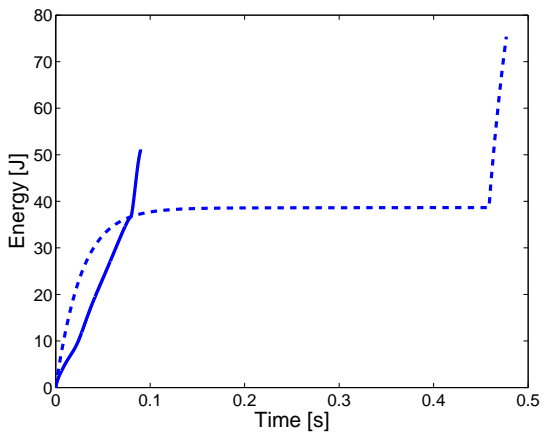

(d)

Figure 10. Performance comparison between first joint of the Silo-4 Legged Robot (dashed lines) and Dual Smart Drive (solid lines).

Lastly, the performance of the Dual Smart Drive was examined in comparison with that of other nonlinear actuators that have been proposed for autonomous robots. In (Montes et al., 2004) the power consumption of the nonlinear Smart Drive and a classical drive were compared experimentally under the same working conditions in quasistatic movement, and the Smart actuator demonstrated an average energy savings of $48 \%$ in comparison with the classical drive. In Table III, a comparison between the Dual Smart Drive and its predecessor, the Smart Drive, is conducted. The drives are tuned for optimal leg movement. Note that for body movement, the Dual Smart Drive proved not only to reduce motion time by $54 \%$, but also to reduce energy consumption by $65 \%$.

These results showcase the Dual Smart Drive's performance edge with respect to other actuation systems. Use of this drive could considerably increase energy efficiency and the time of autonomous robot operation. 
Table II. Performance comparison between first joint of the Silo-4 Legged Robot and Dual Smart Drive.

\begin{tabular}{|c|c|c|}
\hline \multicolumn{3}{|c|}{ FIRST REGIME: MOVEMENT OF THE BODY } \\
\hline & JOINT 1 - SILO 4 & DUAL DRIVE \\
\hline VOLTAGE [V] & 30 & 19.2 \\
\hline TIME [S] & 0.478 & 0.1265 \\
\hline ENERGY [J] & 76.8 & 45.25 \\
\hline
\end{tabular}

\begin{tabular}{|c|c|c|}
\hline \multicolumn{3}{|c|}{ SECOND REGIME: MOVEMENT OF THE LEG } \\
\hline & JOINT 1 - SILO 4 & DUAL DRIVE \\
\hline VOLTAGE [V] & 30 & 19.2 \\
\hline TIME [S] & 0.477 & 0.09 \\
\hline ENERGY [J] & 75 & 51 \\
\hline
\end{tabular}

Table III. Performance comparison between Dual Smart Drive and Smart Drive.

\begin{tabular}{|c|c|c|c|c|}
\hline DRIVE & REGIME & $\begin{array}{c}\text { PART IN } \\
\text { MOVEMENT }\end{array}$ & TIME [S] & ENERGY[J] \\
\hline SMART & -------- & LEG & 0.17 & 1.1 \\
\hline DUAL SMART & SECOND & LEG & 0.17 & 1.1 \\
\hline SMART & -------- & BODY & 2.68 & 87 \\
\hline DUAL SMART & FIRST & BODY & 1.23 & 30 \\
\hline
\end{tabular}




\subsection{Tracking Control Performance}

To investigate the effectiveness of the proposed controllers, several sets of simulations were carried out. The objective was to force the Dual Smart Nonlinear Drive to track the reference trajectories derived with time-optimal control (20) using control laws (30) and (36). The values of the system parameters are given in Table IV. Inertias $J_{1}$ and $J_{2}$ were

Table IV. System parameters.

\begin{tabular}{|c|c|c|c|}
\hline Parameters & Values & Parameters & Values \\
\hline$\ell_{C}$ & $0.065 \mathrm{~m}$ & $J_{M}$ & $0.00011 \mathrm{Kgm}^{2}$ \\
$\ell_{M L}$ & $0.11 \mathrm{~m}$ & $M_{F R i}$ & $0.1 \mathrm{Nm}$ \\
$b_{i}$ & $0.01 \mathrm{Nms}$ & $K_{G}$ & 66 \\
$b_{M 1}$ & $0 \mathrm{Nms}$ & $K_{m}$ & $0.06 \mathrm{Nm} / \mathrm{A}$ \\
$J_{1}$ & $0.0946 \mathrm{Kgm}^{2}$ & $R_{M}$ & $0.6 \Omega$ \\
$J_{2}$ & $0.0046 \mathrm{Kgm}^{2}$ & $K_{e}$ & $0.0425 \mathrm{Vs} / \mathrm{rad}$ \\
\hline
\end{tabular}

chosen to simulate the motion of a robot's body in the first regime and the motion of a robot's leg in the second regime. The initial conditions for the Dual Smart Drive were $\left(x_{1 i}, x_{2 i}\right)=(-145.4 \mathrm{rad}, 0 \mathrm{rad} / \mathrm{s})$. The time-optimal reference trajectories were obtained using the bang-bang control laws:

$$
\left.\begin{array}{llc}
u_{1}^{*}=10.8 \mathrm{~V} & \text { for } & 0 \mathrm{~s}<t \leq 1.165 \mathrm{~s} \\
u_{1}^{*}=-10.8 \mathrm{~V} & \text { for } & 1.165 \mathrm{~s}<t \leq 1.183 \mathrm{~s}
\end{array}\right\} \text { first regime }
$$

The bang-bang components were restricted to values below full actuator saturation, in order to reserve some actuator effort for disturbance compensation and for coping with modeling imperfection.

Figure 11 displays the simulation results using controller (30) with $k_{11}=80, k_{21}=80, \kappa_{1}=1$ for the first working regime of the Dual Smart Drive. Dotted lines represent the desired values, and solid lines represent the actual values. The simulated tracking performance is so satisfactory that in many of the graphics it is difficult to distinguish between the controlled signals and the reference signals. Figure 11(a) and Figure 11(b) show the time evolution of the angular position and the angular velocity of the rotor, respectively. Figure 11(c) and Figure 11(d) show the time evolution of the angular position and the angular velocity of the body, respectively. Figure 11(e) depicts the reference and the actual Dual Smart Drive trajectories in the $x y$-plane, and

nRF-JAR-04-034.tex; 28/07/2005; 18:32; p.24 
Figure 11(f) shows the behavior of the control input signal. Figure 12 illustrates the simulation results using controller (36) with $u_{\max }=12 \mathrm{~V}$, $k_{11}=8, \kappa_{1}=0.00008$ for the first working regime of the Dual Smart Drive. Lastly, Figure 13 displays the same set of curves using controller (36) with $u_{\max }=12 \mathrm{~V}, k_{12}=16, \kappa_{2}=0.0001$ for the second working regime of the Dual Smart Drive. Clearly, all simulation results show that the control objectives were accomplished.

To corroborate the good tracking performance obtained in simulations, different experiments were conducted using the Dual Smart Drive prototype shown in Figure 14. Actual system parameters were all the same as those given in the simulations. Pulse-width modulation (PWM) was used to control the voltage delivered to the motor. A 2000-pulse-per-revolution optical encoder was attached to the motor drive to provide angular position feedback to the controller. The control algorithms were implemented directly in a 486 Processor running real-time operating system QNX.

The same set of curves as presented above were obtained for each experiment. Figure 15 shows simulation and experimental results of system (13) obtained using control law (30). Dotted lines represent simulation results obtained with $k_{11}=80, k_{21}=80, \kappa_{1}=1$, and solid lines represent experimental results obtained with $k_{11}=50$, $k_{21}=80, \kappa_{1}=1$, for the first working regime of the Dual Smart Drive. Figure 16 displays simulation and experimental results of system (13) obtained using control law (36) with $u_{\max }=12 \mathrm{~V}, k_{11}=8$, $\kappa_{1}=0.00008$. Dotted lines represent simulation results and solid lines represent experimental results for the first working regime of the Dual Smart Drive. Figure 17 illustrates simulation and experimental results of system (13) obtained using control law (36) with $u_{\max }=12 \mathrm{~V}$. Dotted lines represent simulation results obtained with $k_{12}=16, \kappa_{2}=0.0001$, and solid lines represent experimental results obtained with $k_{12}=8$, $\kappa_{2}=0.0001$, for the second working regime of the Dual Smart Drive. Lastly, Figures 18 and 19 serve to illustrate the behavior of control laws (30) and (36), respectively, in the presence of a disturbance voltage taking place at $0.3 \mathrm{~s} \leq t \leq 0.35 \mathrm{~s}$.

\subsection{Discussion}

Autonomous robots must be energy efficient but also produce sufficient torque to reach greater speeds. Ongoing research with the Dual Smart Drive therefore attempts to introduce a significant improvement in legged robots and other related mechatronic devices. Subsection 6.1 demonstrated the noteworthy advantages of this drive through simulations and experimental trials. On the other hand, the added 
complexity of the control system is a manifest disadvantage that could restrict the use of this drive. Because of that fact, the simulations and experimental results of the proposed control algorithms are very important from the standpoint of practical implementation. In all the simulations and experiments presented in subsection 6.2, very good tracking performance was obtained with a reasonable control effort. While higher values of the gain $k_{1 i}$ ensured close tracking of the reference in simulations, during the experiments it was found that increasing the gain allows the noise to excite the high-frequency modes of the system and can lead to instabilities. So, it was preferable to use lower values of $k_{1 i}$ in order to make the system less noisesensitive, at the expense of reducing the convergence rate. It was also observed that the first state variable (position) comes very close to the desired level almost immediately, while the second state variable (velocity) experiences lengthier transients. It is interesting to point out that despite the tracking errors of the motor, the mobile link tracks its reference trajectory almost perfectly, due to the intrinsic properties of the Dual Smart Drive. Figures 18 and 19 reveal the importance of having some overhead actuation available in order to recover asymptotic tracking when modeling errors or disturbances cause a deviation. Therefore, the proposed control methods for the Dual Smart Drive provide enough stability, even in the presence of noise and significant disturbances.

\section{Conclusions and Future Developments}

A nonlinear actuator, the Dual Smart Drive, which offers a continuously changing transmission ratio and dual properties and could considerably increase the energy efficiency and the time of autonomous robot operation has been presented. Nonlinear tracking controllers that reflect the idealized dynamics of time-optimal control have been introduced for this drive. These controllers have been constructed using a backstepping design procedure and a universal formula for stabilization with bounded controls. Asymptotic stability and the desired tracking performance have been achieved. The limitations of bang-bang control due to modeling inaccuracies and unpredicted disturbances have been alleviated using backstepping. As modeling and timing accuracies approach perfection, the controllers presented here can approach true time-optimal control. Simulation and experimental results have shown the effectiveness of the controllers and demonstrated that the control objectives were accomplished.

nRF-JAR-04-034.tex; 28/07/2005; 18:32; p.26 
Future developments shall investigate the influence of gravity on the dynamic properties of the Dual Smart Drive, in order to enlarge the drive's area of application in autonomous robots.

\section{Acknowledgements}

R. Fernández would like to acknowledge the Spanish Ministry of Education and Science, which funded her research work at IAI-CSIC and also funded her stay at the Center for Control Engineering and Computation, University of California, Santa Barbara. R. Fernández would also like to thank Professor Petar Kokotović and all the other members of the CCEC lab for a worthwhile, motivating, pleasant stay.

\section{References}

Akinfiev, T. 1990. Method of controlling mechanical resonance hand. Pergamon, US Patent Number: 4958113.

Akinfiev, T. and M. Armada. 1998. Resonance and quasi-resonance drive for startstop regime. Pergamon, Proc. 6th International Conference MECHATRONICS' 98, Skovde, Sweden, pp. 91-96.

Akinfiev, T., M. Armada, J. G. Fontaine, and J. P. Louboutin. 1999. Quasi-resonance drive with adaptive control for start-stop regime. Proceedings of the Tenth World Congress on the Theory of Machines and Mechanisms. IFToMM, Oulu University Press, 5:2049-2054.

Akinfiev, T. and M. Armada. 2000. Some ways of increasing of walking machine drives effectiveness. Proceedings of the 3rd International Conference on Climbing and Walking Robots and the Support Technologies for Mobile Machines. Professional Engineering Publishing Limited London, UK, pp. 519-528.

Akinfiev, T., M. Armada and R. Fernández. 2005. Drive for start-stop movements, especially in walking robots, and its control method. Spanish Patent Number: 2195792.

Armada, M., P. Gonzalez de Santos, M. A. Jimenez and M. Prieto. 2003. Application of CLAWAR Machines. The International Journal of Robotics Research. 22(3-4):251-264.

Athans, M. and P. L. Falb. 1966. Optimal Control. McGraw-Hill Book Company, New York.

Bruneau, O., J. P. Louboutin and J. G. Fontaine. 2000. Optimal design of a legwheel hybrid robot actuated by a quasi-resonant system. Proceedings of the 3rd International Conference on Climbing and Walking Robots and the Support Technologies for Mobile Machines. Professional Engineering Publishing Limited London, UK, pp. 551-558.

Budanov, V. 2001. Underactuated leg of the walking machine. Proceedings of the 4th International Conference on Climbing and Walking Robots. Professional Engineering Publishing Limited London, UK, pp. 167-171.

Caballero, R., T. Akinfiev, H. Montes and M. Armada. 2001. On the modelling of smart nonlinear actuator for walking robots. Proceedings of the 
4th International Conference on Climbing and Walking Robots. Professional Engineering Publishing Limited London, UK, pp. 17-38.

Chilikin, M. and A. Sandler. 1981. General Course of Electric Drives. Energoizdat, Moscow.

Fernández, R., T. Akinfiev and M. Armada. 2003. Modelling and control of the dual smart drive. Proceedings of MED'03 - 11th Mediterranean Conference on Control and Automation, Rhodes, Greece.

Fernández, R., T. Akinfiev and M. Armada. 2003. Dual smart drive: analytical solution, simulation and experimental results. Proceedings of the 6th International Conference on Climbing and Walking Robots and the Support Technologies for Mobile Machines. Professional Engineering Publishing Limited London, UK, pp. 309-318.

García, E. and P. González de Santos. 2001. Soft computing techniques for improving foot trajectories in walking machines. Journal of Robotic Systems. 18(7):251-264.

Ingvast, J. and J. Wikander. 2002. A passive load sensitive revolute transmission. Proceedings of the 5th International Conference on Climbing and Walking Robots and the Support Technologies for Mobile Machines. Professional Engineering Publishing Limited London, UK, pp. 603-610.

Isidori, A. 1989. NonLinear Control Systems. Berlin: Springer-Verlag.

Kanellakopoulos, I., P. V. Kokotovic and A. S. Morse. 1991. Systematic design of adaptive controllers for feedback linearizable systems. IEEE Trans. Automat. Contr., 36:1241-1253.

Khalil, H. K. 2002. Nonlinear Systems. New York: Prentice Hall.

Kokotovic, P. V. 1992. The Joy of Feedback: Nonlinear and Adaptive. IEEE Contr. Sys. Mag., 12:7-17.

Krstic, M., I. Kanellakopoulos and P. V. Kokotovic. 1995. Nonlinear and Adaptive Control Design. New York: Wiley.

Lin, Y. and E. Sontag. 1991. A universal formula for stabilization with bounded controls. Systems \& Control Lett., 16:393-397.

Pfeiffer, F., K. Lffler and M. Gienger. 2000. Design aspects of walking machines. Proc. 3rd International Conference on Climbing and Walking Robots. Professional Engineering Publishing Limited London, UK, pp. 17-38.

Meckl, P. H. and W. Seering. 1985. Active damping in a three-axis robotic manipulator. A.S.M.E. Journal of Vibration, Acoustic, Stress, and Reliability in Design, 107:38-46.

Montes, H., L. Pedraza, M. Armada, T. Akinfiev and R. Caballero. 2004. Adding extra sensitivity to the SMART non-linear actuator using sensor fusion. Industrial Robot: An International Journal, 31:179-188.

Roca, J., J. Palacin, J. Bradineras and J. M. Iglesias. 2002. Lightweight leg design for a static biped walking robot. Proceedings of the 5th International Conference on Climbing and Walking Robots and the Support Technologies for Mobile Machines. Professional Engineering Publishing Limited London, UK, pp. 383-390.

Sardin, P., M. Rostami and G. Besonet. 1998. An anthropomorphic biped robot: dynamic concepts and technological design. IEEE Transactions on Systems, Man and Cybernetics. Part A, Vol. 28.

Song, F. and S. M. Smith. 2000. Design of sliding mode fuzzy controllers for an autonomous underwater vehicle without system model. Oceans'2000 MTS/IEEE, pp. 835-840.

Van De Straete, H. and J. De Schutter. 1999. Optimal time varying transmission for servo motor drives. Proceedings of the Tenth World Congress on the Theory of Machines and Mechanisms. IFToMM, Oulu University Press, 5:2055-2062.

nRF-JAR-04-034.tex; 28/07/2005; 18:32; p.28 
Walking Machine Catalogue. http://gate1.fzi.de/ids/public_html/index2.htm.

\section{Author's Vitae}

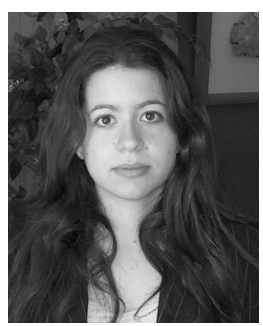

Roemi E. Fernández was born in Madrid, Spain, in 1977. She received the B.S. degree in Electronic Engineering from Santa Maria La Antigua University, Panamá, in 2000. She is currently a Ph.D. candidate at the Polytechnic University of Madrid, Spain and at the Industrial Automation Institute, which belongs to the Spanish Council for Scientific Research. Her research interests include nonlinear control theory, walking and climbing robots, resonance and quasi-resonance drives, and mechatronics.

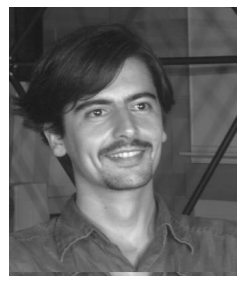

João P. Hespanha was born in Coimbra, Portugal, in 1968. He received the Licenciatura and the M.S. degree in electrical and computer engineering from Instituto Superior Técnico, Lisbon, Portugal, in 1991 and 1993, respectively, and the M.S. and Ph.D. degrees in electrical engineering and applied science from Yale University, New Haven, Connecticut, in 1994 and 1998, respectively. For his PhD work, Dr. Hespanha received Yale University's Henry Prentiss Becton Graduate Prize for exceptional achievement in research in Engineering and Applied Science.

Dr. Hespanha currently holds an Associate Professor position with the Department of Electrical and Computer Engineer at the University of California, Santa Barbara. From 1999 to 2001 he was an Assistant Professor at the University of Southern California, Los Angeles. His 
research interests include switching and hybrid systems; nonlinear control, both robust and adaptive; control of communication networks; the use of vision in feedback control; and stochastic games.

Dr. Hespanha is the recipient of an NSF CAREER Award (2001) and the 2002-2004 Automatica Theory/Methodology best paper prize. Since 2003, he has been an Associate Editor of the IEEE Transactions on Automatic Control.

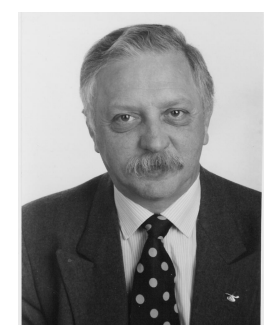

Teodor Akinfiev received his M.S. degree from the Moscow State University and PhD degree from Mechanical Engineering Research Institute of the Academy of Sciences of Russia. From Year 1976 he was Researcher, Principal Researcher and Head of the Research Laboratory at the Mechanical Engineering Research Institute of the Academy of Sciences of Russia. From Year 1995 he holds Position at the Industrial Automation Institute, which belongs to the Spanish Council for Scientific Research. Teodor Akinfiev is the author over 200 publications (including more than 70 patents). His research interests include oscillation theory, mechanical engineering, control systems, robotics, intelligent drives, and mechatronics. In Year 2002 he was elected a Member of the Academy of Natural Sciences of Russia for his research cycle on resonance and quasi-resonance drives.

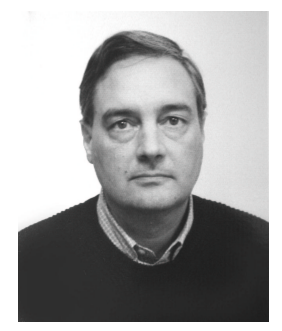

Manuel A. Armada received his $\mathrm{PhD}$ in Physics from the University of Valladolid (Spain) in 1979. Since 1976 he has been involved in 
research activities related to Automatic Control and Robotics. He has been working in more than forty RTD projects (European ones: EUREKA, ESPRIT, BRITE/EURAM, GROWTH, with Latin America: CYTED). He is member of the Russian Academy of Natural Sciences. Dr Armada owns several patents and has published over 200 papers. He is currently the Head of the Automatic Control Department at the Instituto de Automatica Industrial (IAI-CSIC), being his main research in walking and climbing robots. 


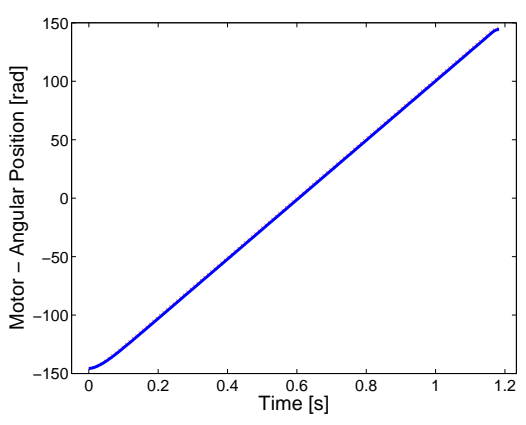

(a)

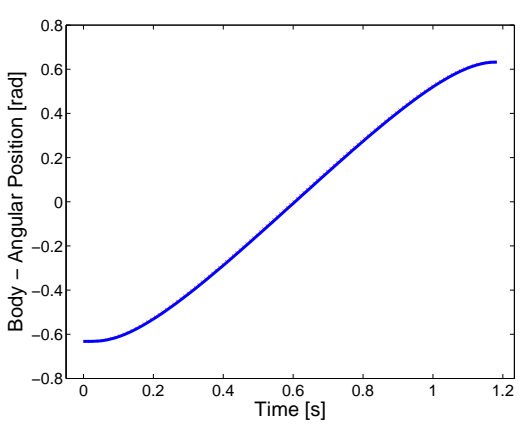

(c)

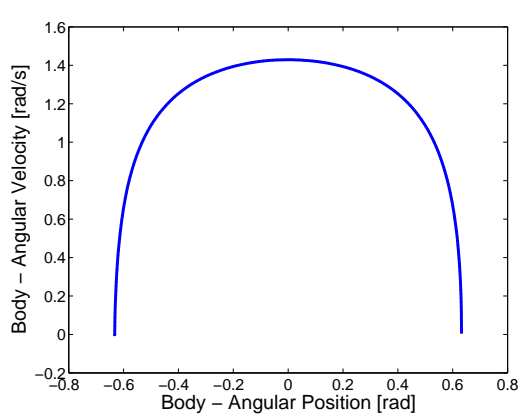

(e)

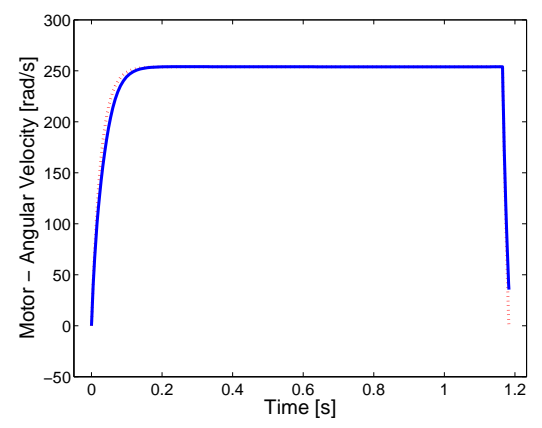

(b)

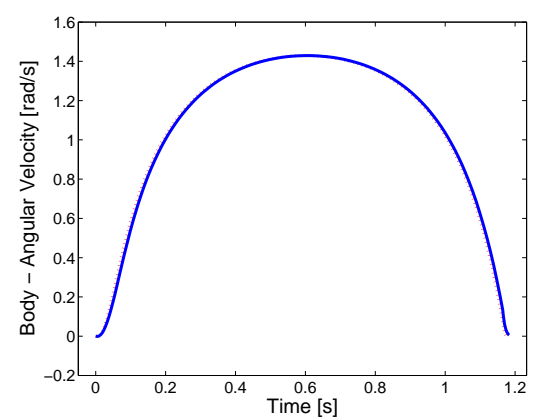

(d)

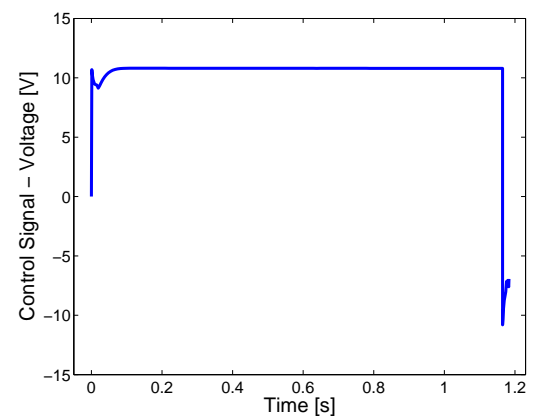

(f)

Figure 11. Simulation results of system (13) obtained using control law (30) with $k_{11}=80, k_{21}=80, \kappa_{1}=1$ for the first working regime of the Dual Smart Drive. In (a)-(e), dotted lines represent the desired signals, and solid lines represent the controlled signals. 


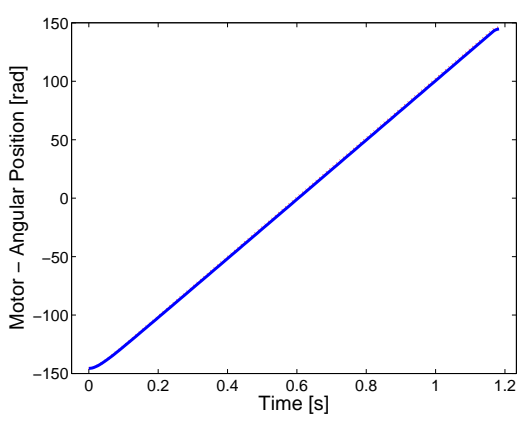

(a)

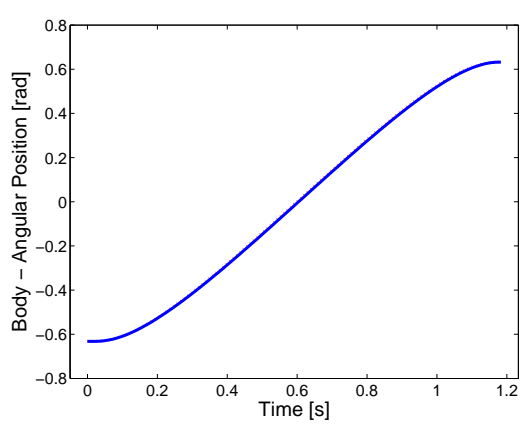

(c)

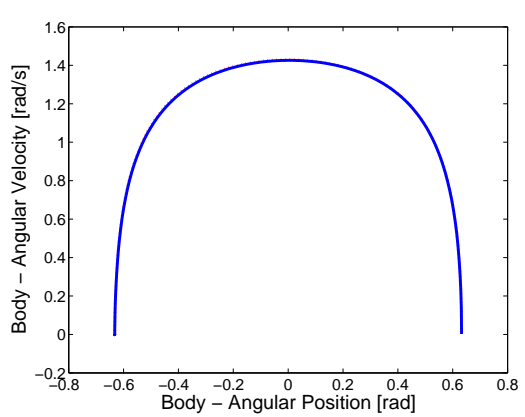

(e)

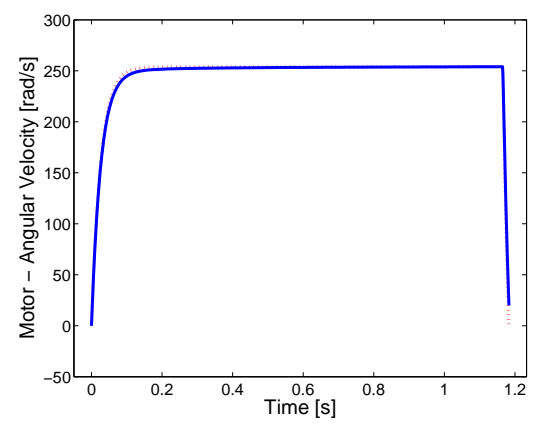

(b)

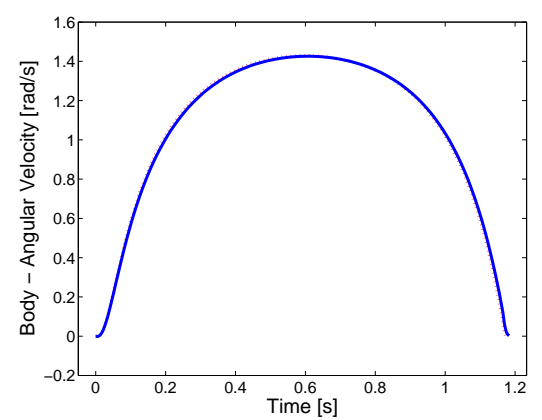

(d)

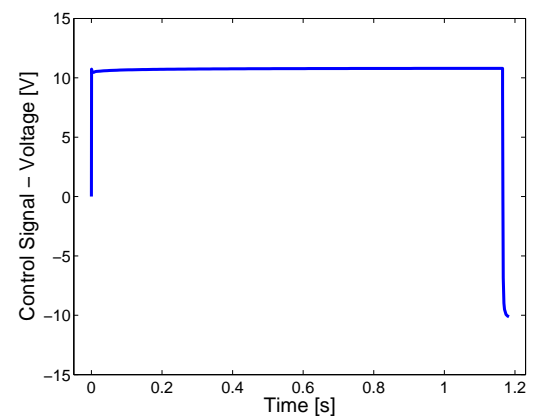

(f)

Figure 12. Simulation results of system (13) obtained using control law (36) with $u_{\max }=12 \mathrm{~V}, k_{11}=8, \kappa_{1}=0.00008$ for the first working regime of the Dual Smart Drive. In (a)-(e), dotted lines represent the desired signals, and solid lines represent the controlled signals. 


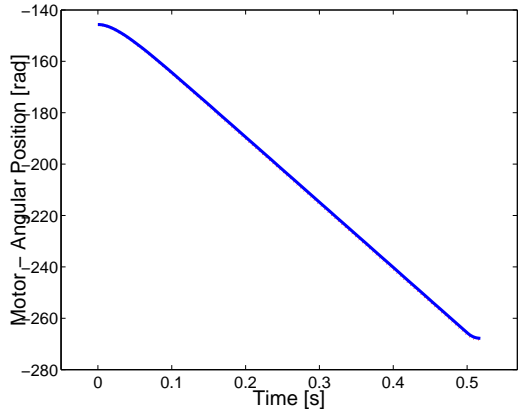

(a)

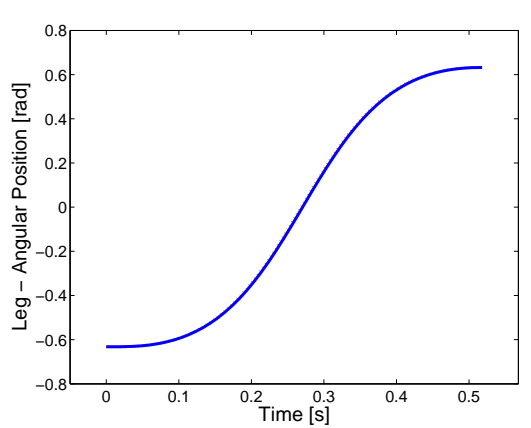

(c)

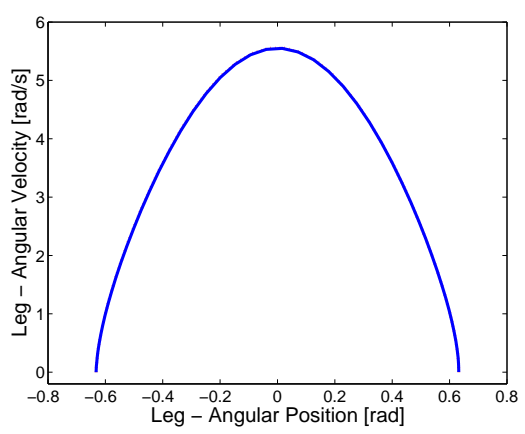

(e)

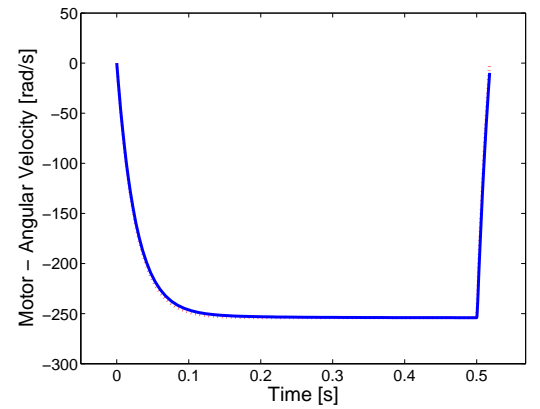

(b)

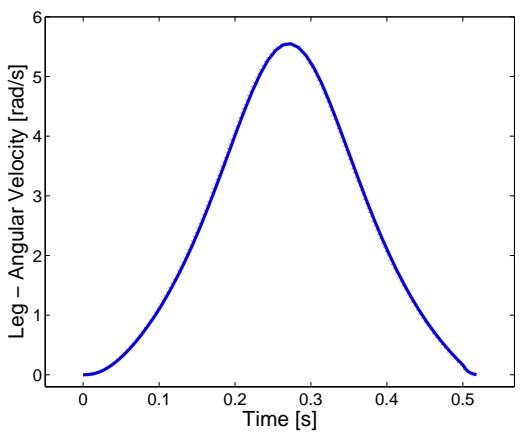

(d)

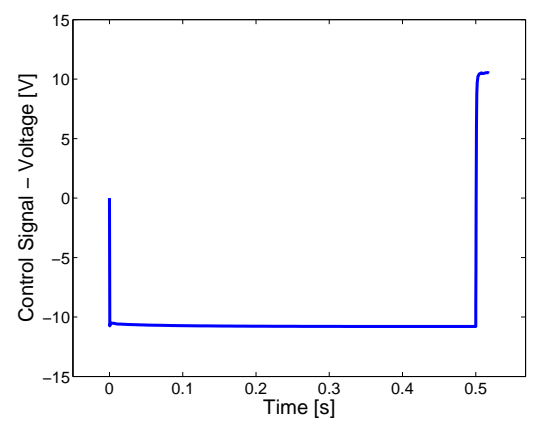

(f)

Figure 13. Simulation results of system (13) obtained using control law (36) with $u_{\max }=12 \mathrm{~V}, k_{12}=16, \kappa_{2}=0.0001$ for the second working regime of the Dual Smart Drive. In (a)-(e), dotted lines represent the desired signals, and solid lines represent the controlled signals. 


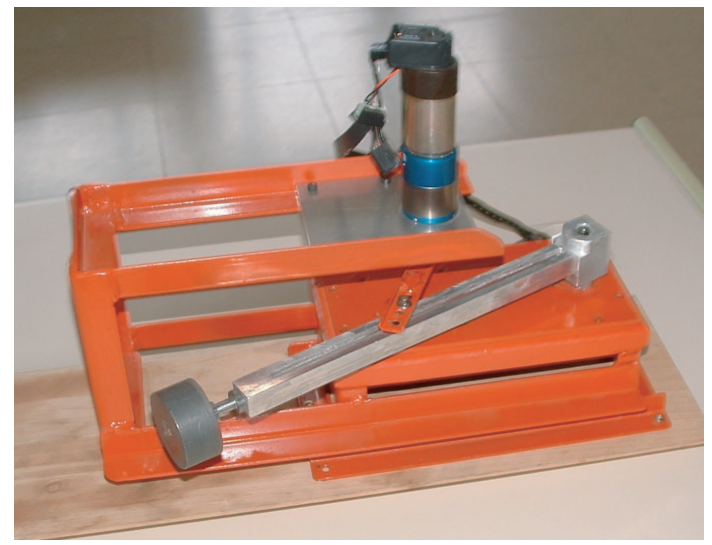

Figure 14. Manufactured prototype of the Dual Smart Drive. 


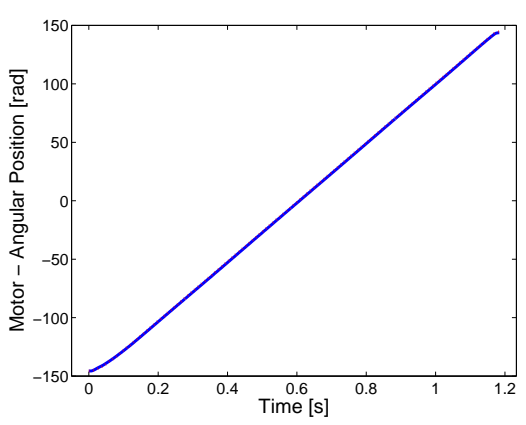

(a)

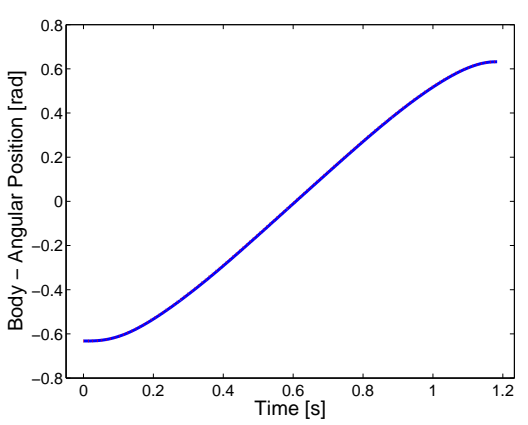

(c)

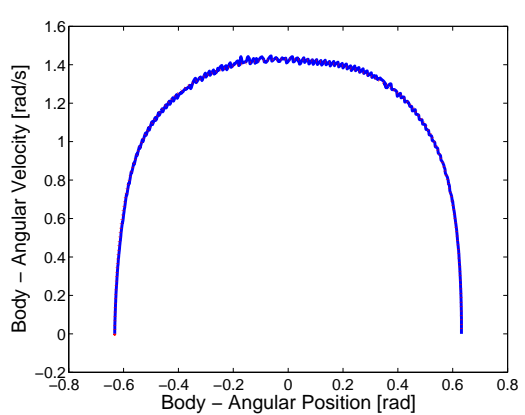

(e)

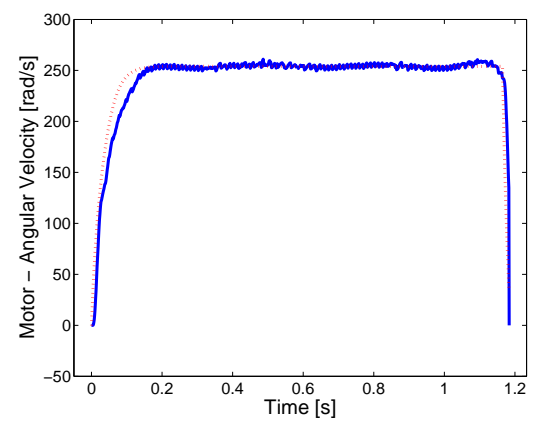

(b)

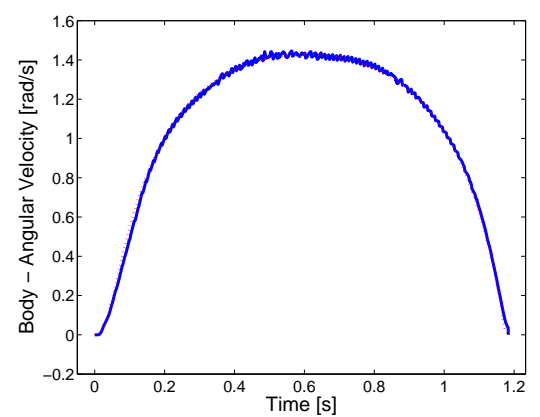

(d)

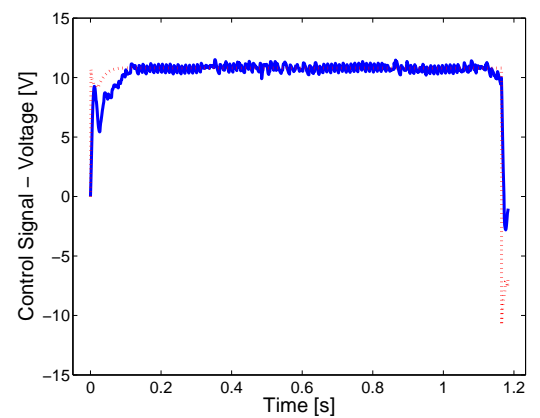

(f)

Figure 15. Simulation and experimental results of system (13) obtained using control law (30). Dotted lines represent simulation results obtained with $k_{11}=80, k_{21}=80$, $\kappa_{1}=1$, and solid lines represent experimental results obtained with $k_{11}=50$, $k_{21}=80, \kappa_{1}=1$, for the first working regime of the Dual Smart Drive. 


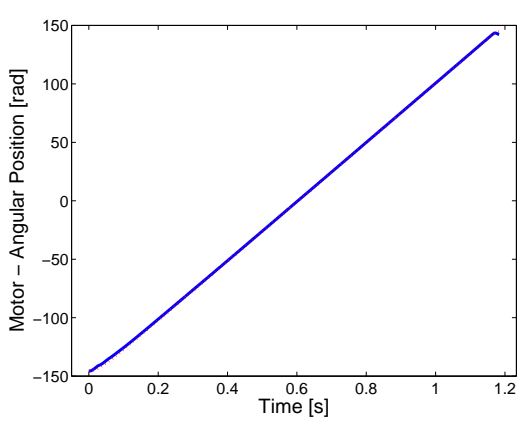

(a)

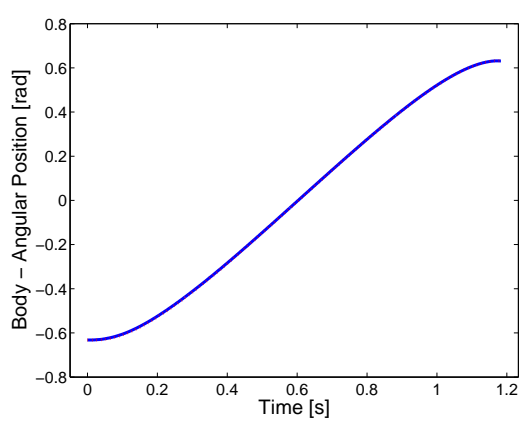

(c)

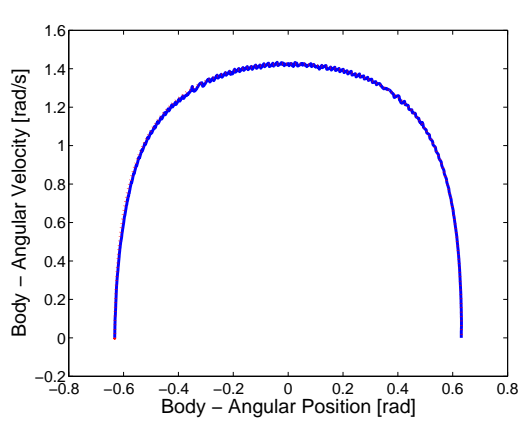

(e)

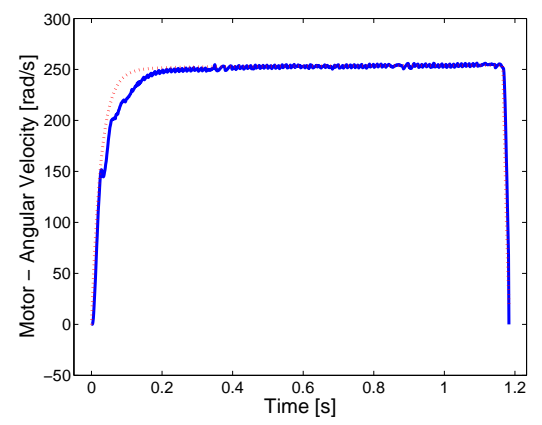

(b)

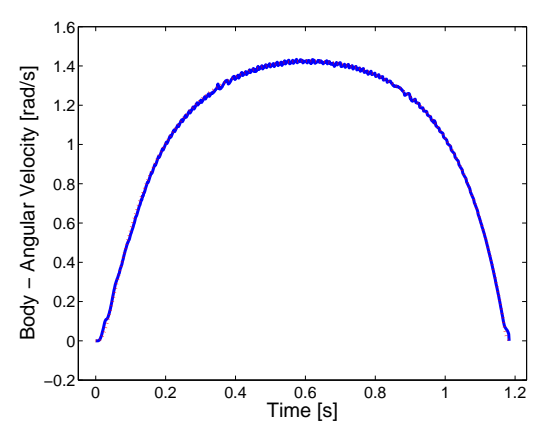

(d)

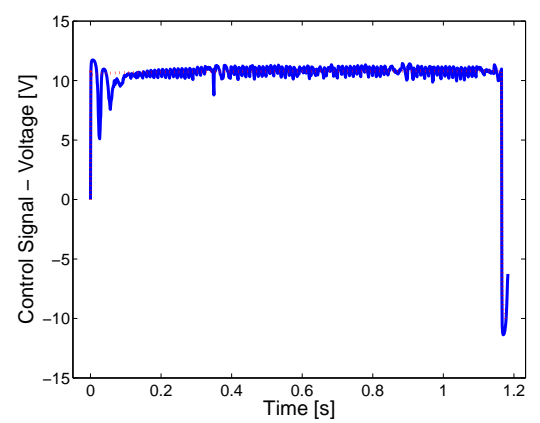

(f)

Figure 16. Simulation and experimental results of system (13) obtained using control law (36) with $u_{\max }=12 \mathrm{~V}, k_{11}=8, \kappa_{1}=0.00008$. Dotted lines represent simulation results and solid lines represent experimental results for the first working regime of the Dual Smart Drive. 


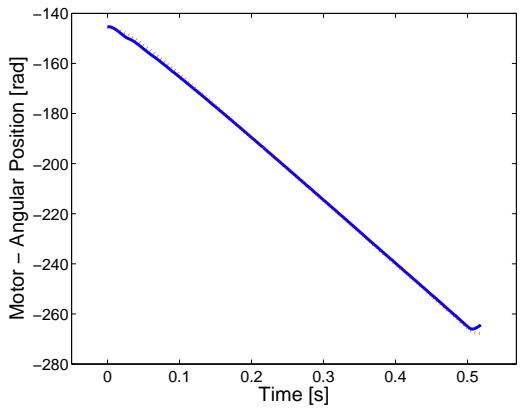

(a)

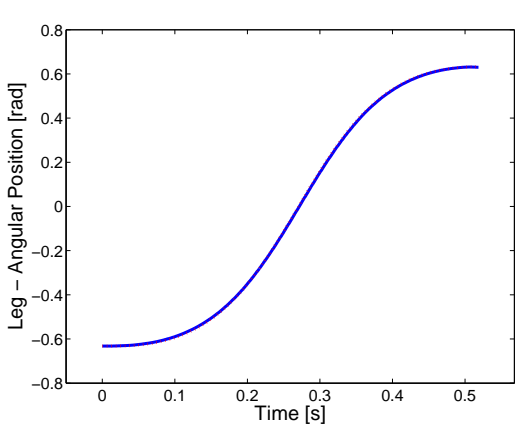

(c)

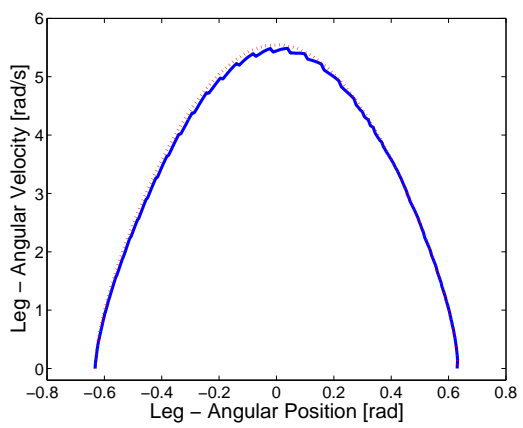

(e)

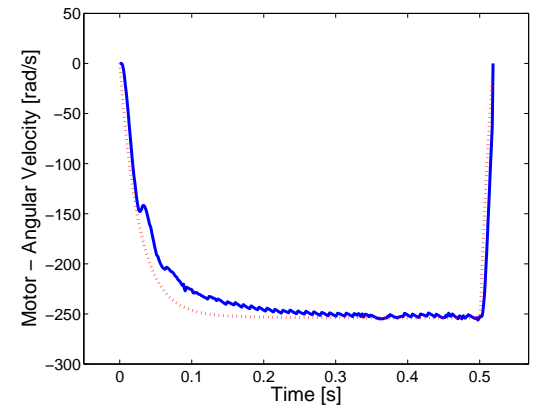

(b)

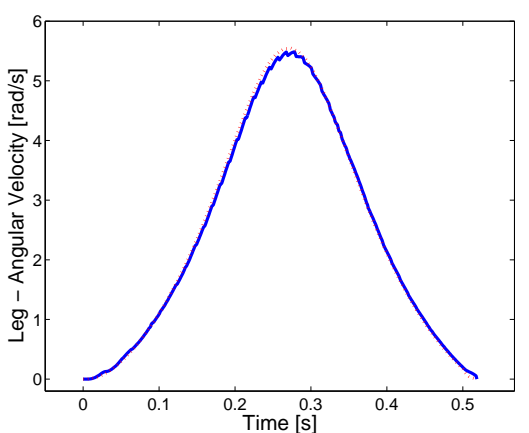

(d)

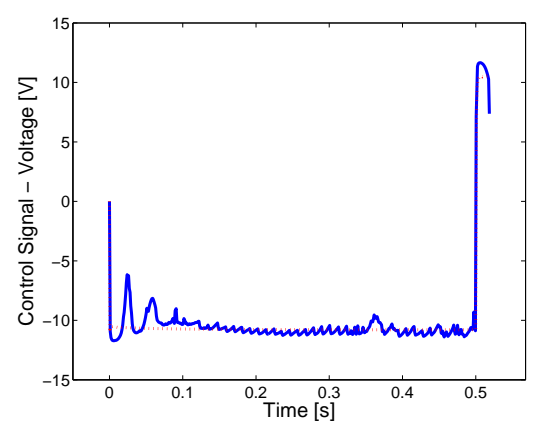

(f)

Figure 17. Simulation and experimental results of system (13) obtained using control law (36) with $u_{\max }=12 \mathrm{~V}$. Dotted lines represent simulation results obtained with $k_{12}=16, \kappa_{2}=0.0001$, and solid lines represent experimental results obtained with $k_{12}=8, \kappa_{2}=0.0001$, for the second working regime of the Dual Smart Drive. 


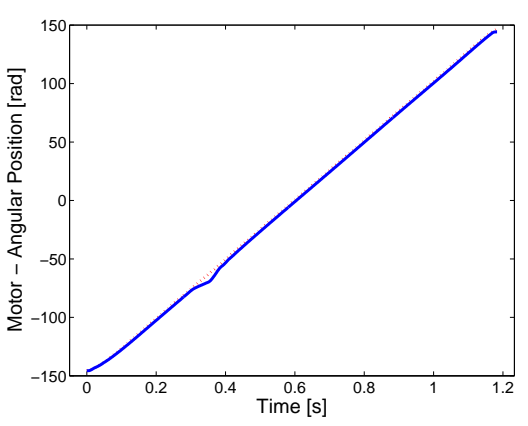

(a)

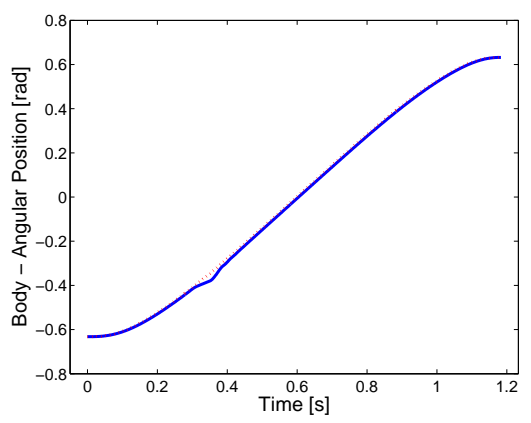

(c)

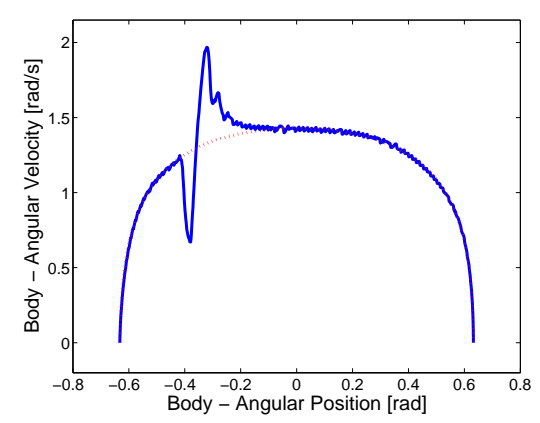

(e)

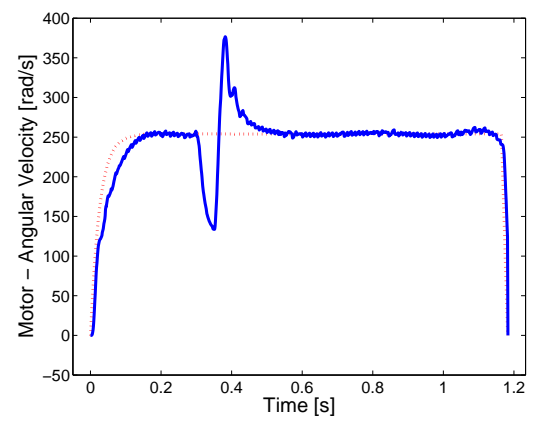

(b)

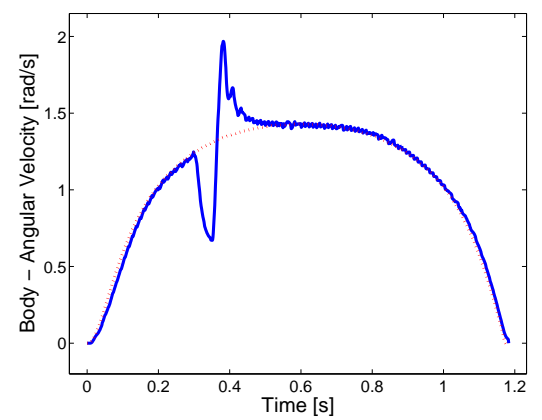

(d)

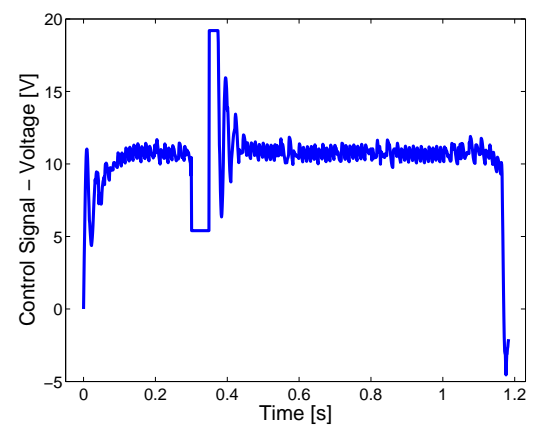

(f)

Figure 18. Experimental results obtained using control law (30) with $k_{11}=50$, $k_{21}=80, \kappa_{1}=1$, for the first working regime of the Dual Smart Drive and with a disturbance at $0.3 \mathrm{~s} \leq t \leq 0.35 \mathrm{~s}$. Dotted lines represent the desired values, and solid lines represent the actual values. 


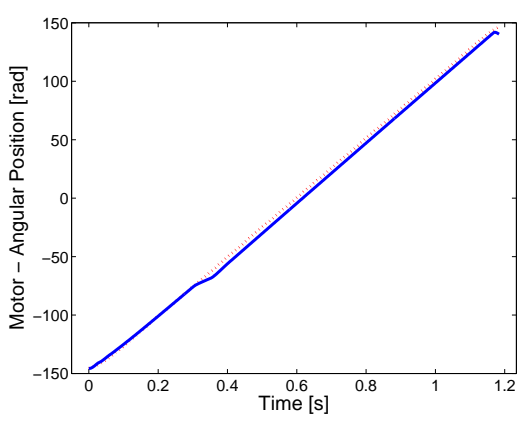

(a)

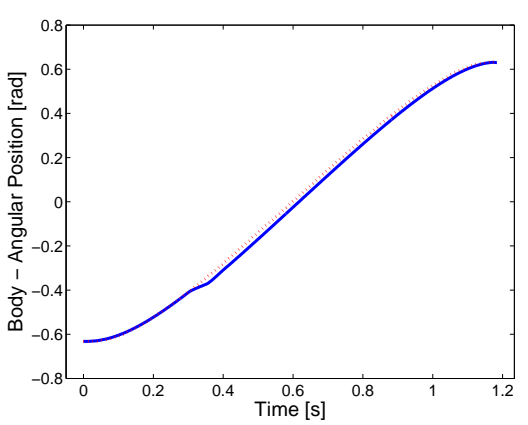

(c)

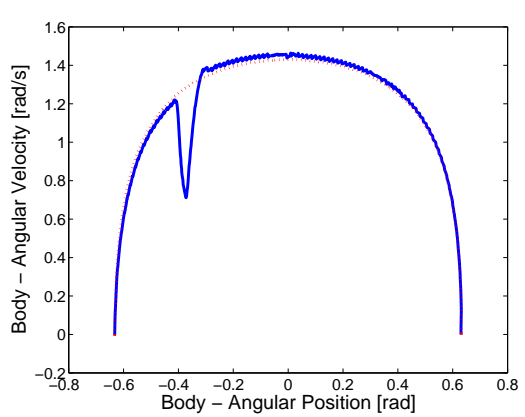

(e)

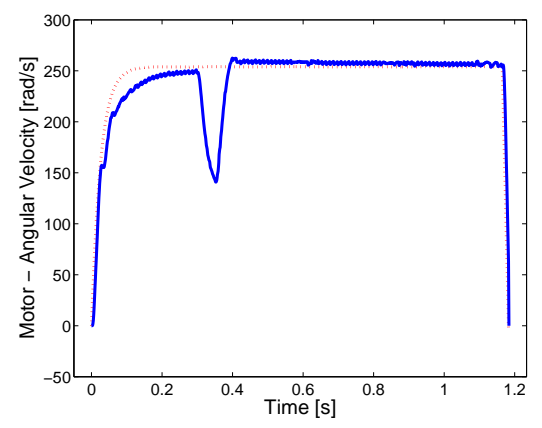

(b)

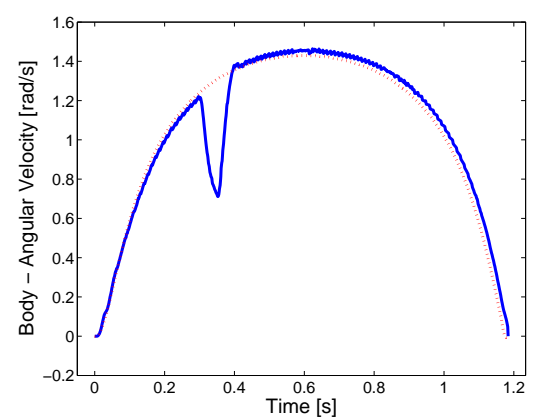

(d)

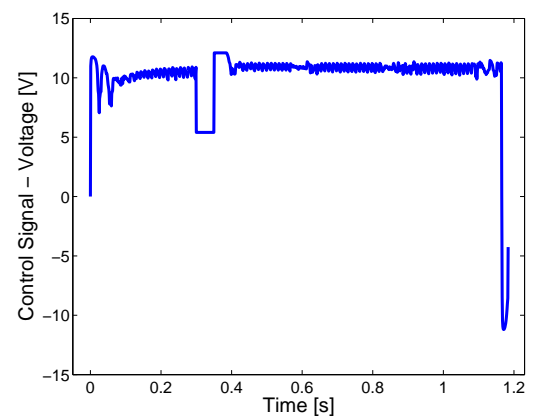

(f)

Figure 19. Experimental results obtained using control law (36) with $u_{\max }=12 \mathrm{~V}$, $k_{11}=8, \kappa_{1}=0.00008$ for the first working regime of the Dual Smart Drive and with a disturbance at $0.3 \mathrm{~s} \leq t \leq 0.35 \mathrm{~s}$. Dotted lines represent the desired values, and solid lines represent the actual values. 